\title{
Medicinal plants and traditional healing practices in ehotile people, around the aby lagoon (eastern littoral of Côte d'Ivoire)
}

\author{
Djah F Malan ${ }^{1,2^{*}}$, Danho F R Neuba ${ }^{1,2}$ and Kouakou L Kouakou ${ }^{1}$
}

\begin{abstract}
Background: Access to useful plants is a growing problem in Africa, increased by the loss of natural vegetation and the erosion of traditional knowledge. Ethnobotany contributes to promote these indigenous knowledge. Despite the large diversity of ethnic groups in Côte d'Ivoire, few ethnomedicine researches have targeted these groups. Among the great Akan group, the Ehotile people are one of the smallest and oldest ethnic group around the Aby Lagoon. The goal of this study was to analyze the level of knowledge and use of medicinal plants by the Ehotile people, and moreover, contribute to build a database about useful plants of first Ivorian people.

Methods: Two sets of surveys were conducted in four Ehotile villages: a house-to-house freelist interview and an individual walk-in-the woods interview with some key informants identified by the community. Frequency of citation, Smith's index, Use value and Informant Consensus Factor were used to estimate the local knowledge of medicinal plants.

Results: Medicinal plants are widely used by Ehotile people. Some were used in addition to modern prescriptions while for some disorders commonly called "African diseases" only plants are used. 123 species employed in the treatment of 57 diseases were listed. Specifically, the most common indications included malaria, sexual asthenia, troubles linked to pregnancy, dysmenorrhoea and haemorrhoids. Analysis of freelists suggested that Ehotile people has a good knowledge of medicinal plants and the most salient included Harungana madagascariensis, Alstonia boonei, Ocimum gratissimum and Xylopia acutiflora. Regarding the consensus among key informants, ICF values were low $(<0.5)$, however category of infectious and parasitic diseases obtained the best agreement $($ ICF $=0.42)$. Following the local experts, 4 types of plants availability were distinguished: Abundant plants easy to collect, abundant plants difficult to harvest, scarce plants and endangered plants.
\end{abstract}

Conclusions: Despite the virtual disappearance of natural formations in Ehotile land, medicinal plants are important in the Ehotile health system. Medicinal plants are known and used alone or in addition to medical prescriptions to treat several ailments. However, some of them are becoming rare, and it is feared that this scarcity will result in the inevitable loss of associated knowledge and practices.

Keywords: Medicinal plants, Traditional knowledge, Ehotile, Côte d'Ivoire

\footnotetext{
* Correspondence: malandfrancois@yahoo.fr

'UFR des Sciences de la Nature, Université Nangui Abrogoua, POB: 02 BP 801, Abidjan 02, République de Côte d'Ivoire

2Institut Botanique Aké-Assi d'Andokoi, POB: 08 BP 172, Abidjan 08, Côte d'Ivoire
}

\section{() Biomed Central}

(c) 2015 Malan et al.; licensee BioMed Central. This is an Open Access article distributed under the terms of the Creative Commons Attribution License (http://creativecommons.org/licenses/by/4.0), which permits unrestricted use, distribution, and reproduction in any medium, provided the original work is properly credited. The Creative Commons Public Domain Dedication waiver (http://creativecommons.org/publicdomain/zero/1.0/) applies to the data made available in this article unless otherwise stated. 


\section{Background}

The contribution of traditional knowledge is invaluable in the long and costly process of developing drug molecules. Thanks to traditional medicine, more than $40 \%$ of commonly prescribed medicines throughout the world are directly or indirectly of natural origin [1]. The use of medicinal plants is still alive in African traditions. Indeed, medicinal plants serve as the primary source of healthcare for the majority of the Developing World's population $[2,3]$. However, access to medicinal plants resources is a growing problem in Africa $[3,4]$. The loss of natural vegetation, due to various causes, is a major factor in the erosion of traditional knowledge.

Ethnobotany, field of Botany that analyzes the results of traditional uses of plants together with the cultural context in which the plants are used [5], contributes by its various approaches to preserve and promote this indigenous knowledge [6].

This approach that emphasizes on a user group (e.g. ethnic group) is not only still relevant [7], but also offers a greater opportunity to gather honest and reliable information in the field.

Many studies, in Africa that are dealing with medicinal uses of plants particularly in Côte d'Ivoire, are simple lists or concern ethnopharmacology. The main focus of studies into traditional pharmacopoeias is the perception of indigenous medicines and the ingredients used in their preparation or the chemical composition, pharmacology and toxicology of plants used [7].

The study of the use of plants, beyond the simple catalogue, using quantitative tools is extremely recent and works discussing the ultimate meaning of disease and the cultural dimension of its treatment are scarce in Côte d'Ivoire. In this country, the large diversity of ethnic groups contrasts with the paucity of ethnomedicine research targeting these groups. Yet, a few reference works [8-10] conducted among Ando, Bete, Abbey and Krobou people and major cross works $[11,12]$ have shown that local specificities are also expressed through medicinal practices. In the complex ethnic mix that characterizes the bank of the Aby Lagoon (eastern littoral of Côte d'Ivoire), the Ehotile people (9 villages) differs from other Akan. The originality of these people is conferred by its territorial precedence, its history and lifestyle closely linked to the lagoon.

Some modern health facilities exist around the Aby lagoon. For example, the four large villages on the west bank of the Aby Lagoon (Etuessika, Melekoukro, N'galwa and Assomlan) have two rural health centres for an estimated 3,500 inhabitants. Each centre is served by one nurse and one midwife, i.e. one health worker for 875 inhabitants. This ratio is certainly significant compared to the Ivorian national ratio, which is 4.8 nurses and midwives per 10,000 inhabitants [13]. However, access to such centres is a major problem for some patients who have to travel a few kilometers or be evacuated by boat.

It's known that when Western medicine is often absent or hard to access or too expensive, the use of remedies from plant becomes particularly significant $[3,14]$. In Ehotile area, this was a fact observed almost on a daily basis. Beside the State health workers, the traditional system of medicine is well established, based on healers whose reputation often goes beyond the borders of the village. For the four villages mentioned above, 15 confirmed healers were registered, i.e. one traditional health agent per 233 inhabitants [15]. The majority of these healers are Komian or acolytes of Komian. The Komian is a priest of traditional religion initiated according to the rites of an order which embodies invisible entities (Boson) whom he worships during public sessions of divination.

Moreover, the land occupied by Ehotile is one of the most degraded of the Ivorian Coast. Scarce natural vegetation that has withstood the plantations of coconut, oil palm or rubber is composed of the marshy patches and the islands of the Ehotile Islands National Park [16]. As the use of plants are often correlated with their availability [17], our study aimed to assess the knowledge and the use of medicinal plants (native or introduced regardless of their medicinal character) in the daily life by Ehotile people, contributing, therefore, to build an integrated database for expertise about useful plants of Ivorian people.

Beyond the study of the use and evaluation of the level of knowledge, our work also aims to probe the availability of the species used with local experts recognized in their community.

\section{Methods}

\section{Study area}

The Ehotile province is composed of 9 main villages round the Aby Lagoon. The survey was conducted in the four biggest, bordering the Ehotile Islands National Park: Mélékoukro, Assomlan (western bank) and Etuoboué and Akounougbé for the eastern bank (Figure 1). The climate of this zone is an equatorial transition type, characterized by four successive seasons: the long rainy season from March to July; the short dry season in August; the small rainy season from September to November and the large less rainy season, from December to February. The average annual rainfalls range from 1800 to $2000 \mathrm{~mm}$ and the annual average temperature is $26.4^{\circ} \mathrm{C}$ with a variation of $3^{\circ} \mathrm{C}$.

The relief of the region is monotonous in the whole with some small plateaus of low altitudes (40 to $60 \mathrm{~m}$ ) with irregular contours, separated by usually quite steep valleys. The soils in this area are generally hydromorphic on Quaternary or young marine sands with in some places, large accumulations of plant debris. 


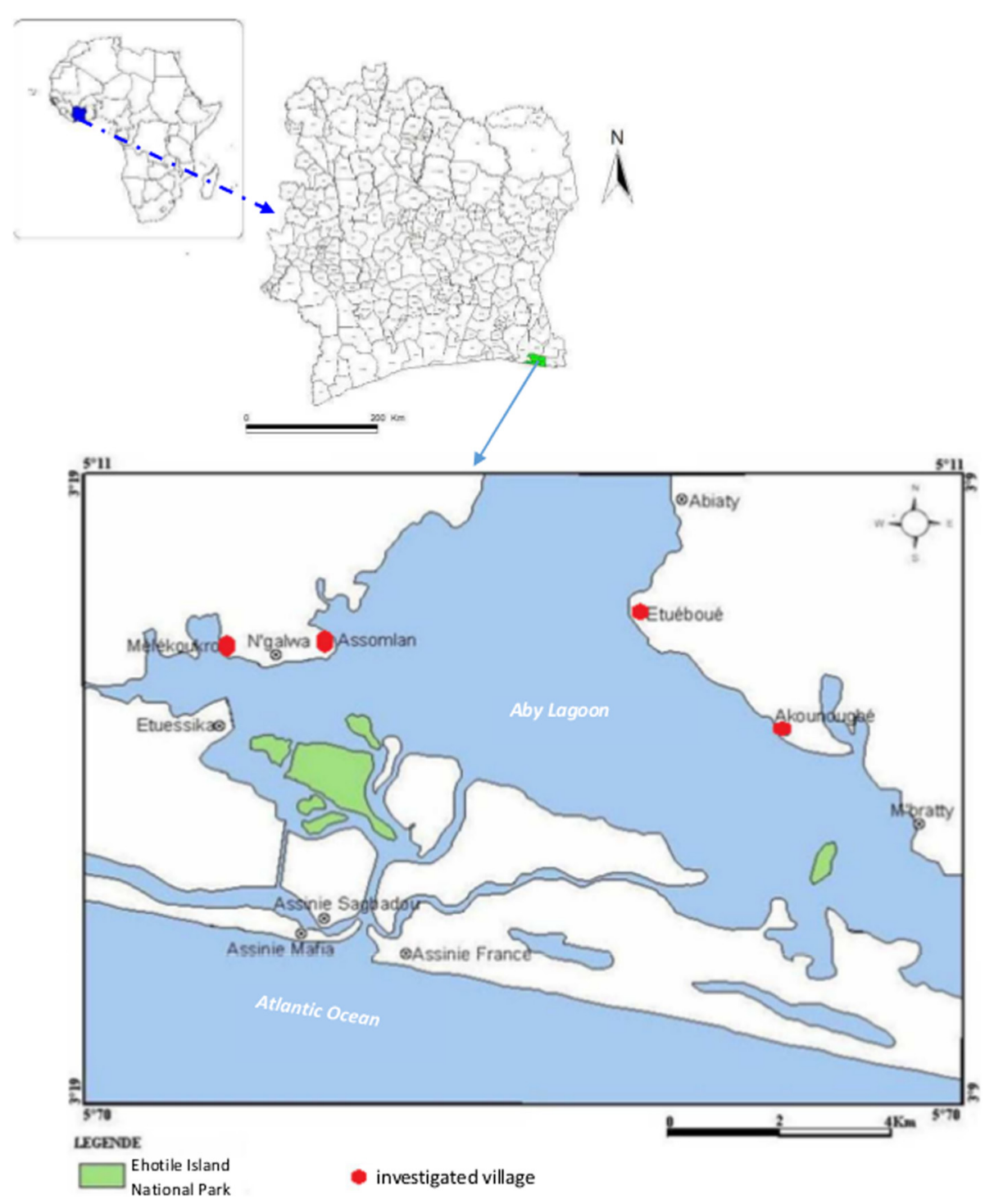

Figure 1 Localisation of study area in Africa, Côte d'Ivoire and around the Aby lagoon.

Concerning the vegetation, the study zone belongs to the Guinean Littoral Area [18]. The original vegetation (Licania eleosperma (Mildbr.) Prance \& White and Drypetes aframensis Hutch. sub-littoral forest) has been replaced by cultivated areas. However, we can distinguish several types of vegetation including mangroves, coastal thickets, forests and plantations. In fact, in this area, due to the variety of soil conditions and trends, there is no dominant climax but a mosaic of edaphic groups.

Fishing is the main activity of the Ehotile people and until very recently (second quarter of the twentieth century), they were exclusively fishermen [19]. Fishing activity (individual or collective) is practiced by men and women. The men are responsible for fishing, the supply of firewood (mangrove wood, mostly) and women are engaged in fish preservation such as, smoking in readiness for marketing. Fishing activities are particularly intense during the long rainy season (May to July) and fall significantly in the dry season (January to April).

Agriculture is the second activity in the study area. From the stage of home consumption with food crops (cassava, yam, banana, etc.) it increased to the stage of cash crop with coconut, oil palm, pineapple and rubber. It is practiced by men and women. Women are mostly engaged in the cultivation of food (cassava and vegetables) while men are the owners of large plantations of coconut, oil palm, rubber or pineapple.

\section{Survey method}

Two sets of surveys were carried out. The first survey was achieved during a house-to-house interview $(\mathrm{N}=55$, 30 women and 25 men, age ranging from 32 to 76 years, 
with an average age of 47.7). Questions were: What are the common diseases here? Do you know and use plants to cure these diseases? Do you know people specialized in traditional treatment of these diseases with plants? Through this step, an initial list of plants used was given and a list of 15 resource persons recognized for their competence in the treatment of various pathologies was established.

The second survey involved some key informants selected among the previous list of identified resource persons $(\mathrm{N}=8,4$ women and 4 men, age ranging from 46 to 70 years, with an average of 60 years). These informed persons were individually interviewed at home (Figure 2) or during a "Walk-in-the-woods" approach. This method involved walking with local people in the areas where they normally collected their medicinal plants while interviewing them [20]. Information on local names of plants and practices was collected. With these key informants who were often reluctant to give their medicinal recipes, our entry point was the availability of resources used. These resource persons followed the dynamics of the surrounding vegetation and knew the common species in their region, those which were rare and those which newly appeared. The collected vouchers were identified at the Herbarium of Ivorian National Floristic Centre (ABJ) and confirmed by the Late Prof. Aké Assi Laurent. Voucher specimens were deposited at the Laboratory of Botany of Nangui Abrogoua University (Abidjan) and at the Institut de Botanique Aké Assi d'Andokoi (Abidjan).

\section{Evaluation of local knowledge}

There are several indices to scientifically estimate indigenous knowledge in a specific field [21]. However, the

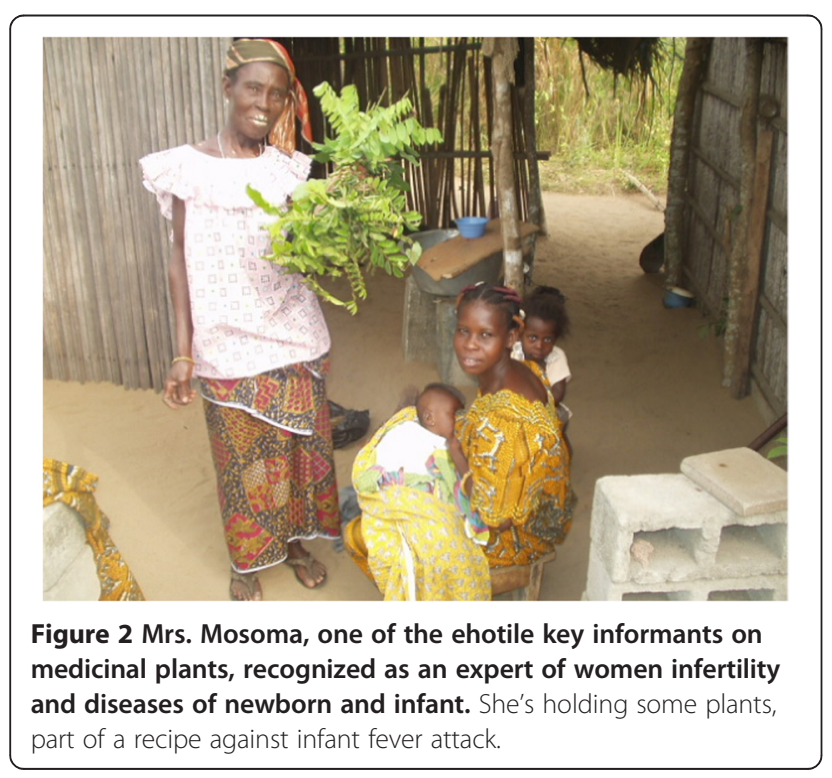

most commonly used are those based on informant consensus, i.e. the level of agreement among various interviewees (see [21-23]). These indices, using spontaneous quotations, are based on the principle that the greater the salience of a given plant or use in the community, the more likely it is to be mentioned [23,24].

One of the simplest and best known is the frequency of citation $(\mathrm{Fq})$, i.e. the number of informants who mentioned a given species. It is a good index to evaluate the credibility of collected information and the level of knowledge within a surveyed population [25]. However, this index does not take into account the rank of appearance of an item in the list of citations. So the Smith's index [25-27] was used. It is based on the frequency of citation, the rank and the length of citation lists and it ranges from 0 to 1 (maximum importance). The formula reviewed [27] is:

$$
S=\left\{\sum\left[\left(L_{i}-R_{j}+1\right) / L_{j}\right]\right\} / N
$$

where $S$ is the salience of an individual item (Smith's index), $L i$ the length of each informant's list, and $R j$ the rank of the item (here, the plant cited) in that list. In addition, the usevalue (UV) was calculated for each plant using the following formula [22,28], modified from [29]:

$$
U V=\sum \frac{U_{i}}{N}
$$

where $U_{i}$ is the number of uses mentioned by each informant for a given species and $\mathrm{N}$ is the total number of informants. Use-value as opposed to the relative importance (RI) greatly emphasizes species that have many uses, even if those uses are known only by a few people [22].

Frequency of citation, Smits's Index and use-value were used for house-to-house data analysis. Furthermore, the Informant Consensus Factor (ICF) was calculated, as follow [29], to assess the agreements among key informants:

$$
I C F=\frac{n_{u r}-n_{t}}{n_{u r}-1}
$$

where $n_{u r}$ is number of use citation in each category and $n_{t}$ number of species used. ICF ranges from 0 (plants are chosen randomly, or informants do not exchange information about their use) to 1 (there is a well-defined selection criterion in the community and/or if information is exchanged between informant $[29,30])$.

Diseases identified were classified into broad categories (considered here as categories of use). The classification was adapted from the $10^{\text {th }}$ version International Statistical Classification of Diseases and related Health Problems or ICD-10 [31]. 


\section{Ethical consideration}

Before being interviewed, local residents of each investigated village were briefed on the research project during an agreement meeting and educated prior informed consent was established following the recommendations of the International Society of Ethnobiology Code of ethics for the publication of this research and any accompanying images. Meetings were held in the village chief's court and the conclusions were sealed, each time with a libation, following the Ehotile customary protocol.

\section{Results}

\section{Observations on Ehotile medicinal practices}

Generally, plants constitute a major part in the health system of Ehotile people. Information on popular medicinal recipes is easily accessible. Among the diseases listed in our work, some are subject to a complementary treatment (modern prescriptions and medicinal plants) including malaria, diarrhoea, and headache. The first treatment is usually provided by the housewives. That is why basic medicinal plants such as Ocimum gratissimum L., Eclipta prostrata (L.) L., Capsicum frutescens L. are grown in the homegardens or backyards in village settings. These plants constitute a kind of living "pharmacy box".

Beside diseases for which complementarity between traditional and modern medicines was observed, there are some disorders commonly called "African-diseases" for which modern medicines are not sought. For these, it is the healer who is solicited. In some cases, the complexity of the described symptoms or their non-organic origin has led to the retention of the local names of these ailments: Asema, elokposan or butumanklan.

Finally, there is a last category of diseases where treatment offered in health centres is not accepted by villagers. These illnesses include haemorrhoids, hydrocephalus or disorders of reproductive health (sexual asthenia, impotence or infertility).

\section{Plants used in common traditional medicine by Ehotile people}

The investigation yielded a list of 123 plant species, 96 after the house-to-house survey and 27 only collected with local experts (Table 1). These taxa were distributed in 113 genera and 52 plant families (44 of Dicotyledons, 6 of Monocotyledons and 2 of Pteridophytes). The richest ones were Leguminosae (15 species), Euphorbiaceae (9), Annonaceae (7), Apocynaceae (6) and Compositae (6).

All morphological types are represented but with a high prevalence of trees and shrubs (61.8\%) followed by lianas $(22.8 \%)$ and herbaceous species (15.4\%). Most of the plants used were native species $(85.3 \%)$ though few exotic species were recorded $(14.7 \%)$. These exotic plants included mango tree (Mangifera indica L.), papaya (Carica papaya L.), guava (Psidium guajava L.), plantain (Musa paradisiaca L.), introduced for their edible fruit and neem tree (Azadirachta indica), introduced as ornamental or shade plant. These plants, over time, play an important role in traditional African medicines.

All the plants listed were used for 59 indications (9 exclusively from key informants, 11 from laypeople and 39 from both groups) sorted in 12 categories (Figure 3 ). The most cited category is the group of infectious and parasitic diseases $(84 \%)$, followed remotely by the diseases of the genital system, pregnancy and childbirth (46\%) and the diseases of the digestive system (34\%). Categories of endocrine, nutritional \& metabolic diseases and diseases of the respiratory system are the least common with only $7 \%$ of the citations.

Specifically, the most common indications included malaria ( $82 \%$ of citations), sexual weakness (29\%), development of pregnancy (23\%), dysmenorrhoea (22\%) and haemorrhoids (21\%).

Analysis of free lists suggested that Ehotile people, in general, has a good knowledge of medicinal plants. The length of the lists ranged from 1 (only one informant) to 38 plants, with an average of 7.1 plants per list. However, frequent lengths were 3 plants per list $(25.5 \%$ of informants) and 4 per list (18.2\% of informants).

Regarding the salience of plants utilized, Harungana madagascariensis Lam. ex Poir. showed the highest Smith's index $(\mathrm{S}=0.38)$. This plant was widely cited $(\mathrm{Fq}=0.53)$ but with an average rank of 3.9 and a low use-value (UV $=0.02)$. H. madagascariensis was followed by Alstonia boonei De Wild. $(\mathrm{S}=0.32 ; \mathrm{Fq}=0.35$, average rank $=1.8$ and $\mathrm{UV}=0.02)$, Ocimum gratissimum $\mathrm{L}$. $(\mathrm{S}=0.24 ; \mathrm{Fq}=0.38$, average rank = 3.57 and UV $=0.13$ ) and Xylopia acutiflora (Dunal) A.Rich. $(\mathrm{S}=0.22 ; \mathrm{Fq}=0.29$, average rank $=3.88$ and $\mathrm{UV}=0.04)$.

Seven types of drugs were listed in very different proportions including leaves (42\%), barks $(25 \%)$, roots and tubers (19\%), fruits and seeds (8\%), whole plant (4\%), inflorescences (1\%) and exudates (1\%). Anal route using enema bag $(33.2 \%)$, oral route $(27.4 \%)$ and body brushing $(13.9 \%)$ constituted the main administration routes.

\section{Key informant consensus and resources availability}

The survey among specialists permitted to collect 92 species of which 27 are specific to this group, for 48 therapeutic indications. These specific plants included Antiaris toxicaria Lesch. Blighia welwitschii (Hiern) Radlk., Chassalia kolly (Schumach.) Hepper, Costus afer Ker Gawl., Diospyros sanza-minika A.Chev., Euadenia trifoliolata (Schumach. \& Thonn.) Oliv., Mareya micrantha (Benth.) Müll. Arg., Microgramma owariensis (Desv.) Alston, Vernonia conferta Benth., Plumbago zeylanica L., Ocimum americanum L. $(\mathrm{Fq}=1)$, Icacina mannii Oliv. (Fq = 0.75), Uvaria afzelii G.F. Scott-Elliot $(\mathrm{Fq}=0.75)$, Zanthoxylum gilletii (De Wild.) P.G. 
Table 1 List of medicinal plants used by Ehotile people

\begin{tabular}{|c|c|c|c|c|c|c|c|}
\hline Species & $\begin{array}{l}\text { Voucher } \\
\text { number }\end{array}$ & Family & Informant & $\mathrm{Fq}$ & $\begin{array}{l}\text { Smiths' } \\
\text { S }\end{array}$ & Indication & $\begin{array}{l}\text { Part used and administration } \\
\text { mode }\end{array}$ \\
\hline \multirow[t]{2}{*}{ Abrus precatorius L. } & \multirow[t]{2}{*}{ Malan 758} & \multirow[t]{2}{*}{ Leguminosae } & \multirow[t]{2}{*}{ LP } & \multirow[t]{2}{*}{0.04} & \multirow[t]{2}{*}{0.015} & Ophtalmia & Eye drops with leaf juice \\
\hline & & & & & & Palpitations & Leaf juice swallowed \\
\hline $\begin{array}{l}\text { Adenia lobata (Jacq.) } \\
\text { Engl. }\end{array}$ & Malan 504 & Passifloraceae & $L P$ & 0.04 & 0.032 & Ophtalmia & Eye drops with sap \\
\hline Ageratum conyzoides L. & AD 128 & Compositae & $L P$ & 0.04 & 0.016 & Headache & Nose drops with leaf juice \\
\hline $\begin{array}{l}\text { Albertisia cordifolia } \\
\text { (Mangenot \& Miège) } \\
\text { Forman }\end{array}$ & $\begin{array}{l}\text { Malan 638, } \\
718\end{array}$ & Menispermaceae & $L P, K l$ & 0.07 & 0.04 & Oedema & $\begin{array}{l}\text { Topical application with } \\
\text { crushed leaves }\end{array}$ \\
\hline $\begin{array}{l}\text { Albizia zygia } \\
\text { (DC.) J. F. Macbr. }\end{array}$ & Malan 596 & Leguminosae & $L P, K l$ & 0.02 & 0.018 & Rheumatism & $\begin{array}{l}\text { Topical application with } \\
\text { crushed leaves }\end{array}$ \\
\hline \multirow{4}{*}{$\begin{array}{l}\text { Alchornea cordifolia } \\
\text { (Schumach. \& Thonn.) } \\
\text { Müll.Arg. }\end{array}$} & \multirow[t]{4}{*}{ AD 224} & \multirow[t]{4}{*}{ Euphorbiaceae } & \multirow[t]{4}{*}{$L P, K I$} & \multirow[t]{4}{*}{0.05} & \multirow[t]{4}{*}{0.047} & White discharge & $\begin{array}{l}\text { Enema with leaves mashed } \\
\text { and diluted in water }\end{array}$ \\
\hline & & & & & & Malaria & $\begin{array}{l}\text { Leaves decoction drunk and } \\
\text { taken as enema }\end{array}$ \\
\hline & & & & & & Wound & $\begin{array}{l}\text { Topical application with } \\
\text { crushed leaves }\end{array}$ \\
\hline & & & & & & Sexual asthenia & Leaves decoction drunk \\
\hline $\begin{array}{l}\text { Alstonia boonei } \\
\text { (DC.) Willd. }\end{array}$ & Malan 630 & Apocynaceae & $L P, K I$ & 0.35 & 0.294 & Malaria & $\begin{array}{l}\text { Leaves and bark decoction } \\
\text { drunk and taken as enema }\end{array}$ \\
\hline \multirow[t]{2}{*}{$\begin{array}{l}\text { Alternanthera } \\
\text { pungens Kunth }\end{array}$} & \multirow[t]{2}{*}{ AD 132} & \multirow[t]{2}{*}{ Amaranthaceae } & \multirow[t]{2}{*}{$L P, K I$} & \multirow[t]{2}{*}{0.05} & \multirow[t]{2}{*}{0.038} & Convulsion & $\begin{array}{l}\text { Body smeared with leaves } \\
\text { mashed }\end{array}$ \\
\hline & & & & & & Infant diarrhoea & $\begin{array}{l}\text { Enema with leaves mashed } \\
\text { and diluted in water }\end{array}$ \\
\hline \multirow{2}{*}{$\begin{array}{l}\text { Anchomanes difformis } \\
\text { (Blume) Engl. }\end{array}$} & \multirow[t]{2}{*}{ Malan 570} & \multirow[t]{2}{*}{ Araceae } & \multirow[t]{2}{*}{ LP } & \multirow[t]{2}{*}{0.04} & \multirow[t]{2}{*}{0.023} & Dysmenorrhoea & \multirow{2}{*}{$\begin{array}{l}\text { Enema with tuber mashed } \\
\text { and diluted in water }\end{array}$} \\
\hline & & & & & & Pregnancy care & \\
\hline \multirow[t]{2}{*}{$\begin{array}{l}\text { Anthocleista nobilis } \\
\text { G.Don }\end{array}$} & \multirow[t]{2}{*}{ AD 63} & \multirow[t]{2}{*}{ Gentianaceae } & \multirow[t]{2}{*}{$L P, K l$} & 0.04 & 0.034 & Malaria & $\begin{array}{l}\text { Body smeared with mashed } \\
\text { bark }\end{array}$ \\
\hline & & & & & & Rheumatism & $\begin{array}{l}\text { Topical application with } \\
\text { crushed roots }\end{array}$ \\
\hline $\begin{array}{l}\text { Antiaris toxicaria } \\
\text { (Engl.) C.C. Berg }\end{array}$ & Malan 600 & Moraceae & $L P, K I$ & 0.04 & 0.016 & Hydrocephalus & Bathing with bark decoction \\
\hline $\begin{array}{l}\text { Azadirachta indica } \\
\text { A.Juss. }\end{array}$ & & Meliaceae & $L P, K I$ & 0.07 & 0.053 & Malaria & $\begin{array}{l}\text { Body smeared with mashed } \\
\text { leaves, leaves decoction drunk }\end{array}$ \\
\hline $\begin{array}{l}\text { Bambusa vulgaris } \\
\text { Wendel. ex Nees }\end{array}$ & Malan 447 & Poaceae & $L P, K I$ & 0.09 & 0.06 & Malaria & Leaves decoction drunk \\
\hline Baphia nitida Lodd. & AD 166 & Leguminosae & $L P, K I$ & 0.11 & 0.08 & Bubo & $\begin{array}{l}\text { Topical application with } \\
\text { crushed leaves }\end{array}$ \\
\hline & & & & & & Bad breath & Roots as chewing stick \\
\hline & & & & & & Boil & $\begin{array}{l}\text { Topical application with } \\
\text { crushed leaves }\end{array}$ \\
\hline & & & & & & Lumbar pain & $\begin{array}{l}\text { Enema with mashed roots } \\
\text { diluted in water }\end{array}$ \\
\hline Bidens pilosa L. & AD 112 & Compositae & $L P, K I$ & 0.09 & 0.061 & Hydrocephalus & Body smeared with mashed \\
\hline & & & & & & Diarrhoea & $\begin{array}{l}\text { leaves Enema with leaves } \\
\text { mashed and diluted in water }\end{array}$ \\
\hline & & & & & & Malaria & \\
\hline & & & & & & Giddiness & \\
\hline Blighia sapida Koenig & AD 196 & Sapindaceae & $L P$ & 0.04 & 0.026 & Lumbar pain & Enema with bark mashed and \\
\hline & & & & & & Malaria & \\
\hline & & & & & & Rheumatism & $\begin{array}{l}\text { Topical application with } \\
\text { crushed bark }\end{array}$ \\
\hline
\end{tabular}


Table 1 List of medicinal plants used by Ehotile people (Continued)

\begin{tabular}{|c|c|c|c|c|c|c|c|}
\hline \multirow[t]{2}{*}{$\begin{array}{l}\text { Blighia welwitschii } \\
\text { (Hiern) Radlk. }\end{array}$} & \multirow[t]{2}{*}{ Malan 968} & \multirow[t]{2}{*}{ Sapindaceae } & \multirow[t]{2}{*}{$\mathrm{KI}$} & \multirow[t]{2}{*}{-} & \multirow[t]{2}{*}{-} & $\begin{array}{l}\text { Malaria } \\
\text { Rheumatism }\end{array}$ & \multirow[t]{2}{*}{$\begin{array}{l}\text { Enema with bark mashed and } \\
\text { diluted in water Topical } \\
\text { application with crushed bark }\end{array}$} \\
\hline & & & & & & Oedema & \\
\hline $\begin{array}{l}\text { Bridelia micrantha } \\
\text { (Hochst.) Baill. }\end{array}$ & AD 89 & Phyllanthaceae & LP & 0.04 & 0.031 & Malaria & $\begin{array}{l}\text { Enema with leaves mashed } \\
\text { and diluted in water }\end{array}$ \\
\hline \multirow{2}{*}{$\begin{array}{l}\text { Caesalpinia bonduc } \\
\text { (L.) Roxb. }\end{array}$} & \multirow[t]{2}{*}{ AD 240} & \multirow[t]{2}{*}{ Leguminosae } & \multirow[t]{2}{*}{$L P, K I$} & \multirow[t]{2}{*}{0.05} & \multirow[t]{2}{*}{0.045} & Malaria & \multirow{2}{*}{$\begin{array}{l}\text { Enema with leaves mashed } \\
\text { and diluted in water }\end{array}$} \\
\hline & & & & & & Sexual asthenia & \\
\hline Cajanus cajan (L.) Roxb. & \multirow[t]{4}{*}{ AD 13} & Leguminosae & $L P, K I$ & 0.02 & 0.012 & Malaria & Bathing with leaves decoction \\
\hline \multirow[t]{2}{*}{ Capsicum frutescens $\mathrm{L}$. } & & \multirow[t]{2}{*}{ Solanaceae } & \multirow[t]{2}{*}{$\mathrm{Kl}$} & \multirow[t]{2}{*}{0.04} & \multirow[t]{2}{*}{0.018} & $\begin{array}{l}\text { Serious disease of } \\
\text { unknown origin }\end{array}$ & $\begin{array}{l}\text { Ocular instillations with } \\
\text { leaf juice }\end{array}$ \\
\hline & & & & & & Contraception & $\begin{array}{l}\text { Enema with fruits mashed } \\
\text { and diluted in water }\end{array}$ \\
\hline & & Caricaceae & $L P, K I$ & 0.05 & 0.028 & Malaria & Leaves decoction drunk \\
\hline Carpolobia lutea G.Don & AD07 & Polygalaceae & $L P, K I$ & 0.05 & 0.037 & Sexual asthenia & Roots decoction drunk \\
\hline \multirow[t]{3}{*}{ Cassia alata L. } & \multirow[t]{3}{*}{ AD 15} & \multirow[t]{3}{*}{ Leguminosae } & \multirow[t]{3}{*}{$L P, K I$} & 0.18 & 0.1 & Dry patch & $\begin{array}{l}\text { Topical application with } \\
\text { crushed leaves }\end{array}$ \\
\hline & & & & & & Malaria & $\begin{array}{l}\text { Body smeared with leaves } \\
\text { mashed }\end{array}$ \\
\hline & & & & & & Constipation & Leaves decoction drunk \\
\hline Cassia occidentalis L. & Malan 618 & Leguminosae & $L P, K I$ & 0.07 & 0.04 & Headache & Nose drops with leaf juice \\
\hline & & & & & & Malaria & Leaves decoction drunk \\
\hline $\begin{array}{l}\text { Catharanthus roseus } \\
\text { (L.) G.Don }\end{array}$ & & Apocynaceae & $L P$ & 0.02 & 0.015 & Inguinal hernia & $\begin{array}{l}\text { Enema with mashed roots } \\
\text { diluted in water }\end{array}$ \\
\hline $\begin{array}{l}\text { Chassalia koly } \\
\text { (Schumach.) Hepper }\end{array}$ & Malan 717 & Rubiaceae & $L P, K I$ & 0.02 & 0.011 & Headache & Eye drops with juice from roots \\
\hline $\begin{array}{l}\text { Cleistopholis patens } \\
\text { (Benth.) Engl. \& Diels }\end{array}$ & AD 68 & Annonaceae & $L P, K I$ & 0.11 & 0.07 & $\begin{array}{l}\text { Rheumatism } \\
\text { Elokposan }\end{array}$ & $\begin{array}{l}\text { Topical application with } \\
\text { crushed bark }\end{array}$ \\
\hline Cnestis ferruginea & Malan 395 & Connaraceae & $L P, K I$ & 0.05 & 0.037 & Hydrocephalus & Bathing with leaves decoction \\
\hline vani ex D. & & & & & & Pregnancy care & $\begin{array}{l}\text { Enema with roots mashed and } \\
\text { diluted in water }\end{array}$ \\
\hline Cocos nucifera L. & & Arecaceae & $L P$ & 0.02 & 0.017 & Raging toothache & $\begin{array}{l}\text { Mouthwash with roots } \\
\text { decoction }\end{array}$ \\
\hline & & & & & & Butumanklan & $\begin{array}{l}\text { Enema with roots mashed and } \\
\text { diluted in water }\end{array}$ \\
\hline $\begin{array}{l}\text { Cola lateritia var. maclaudi } \\
\text { (A.Chev.) Brenan \& Keay }\end{array}$ & $\begin{array}{l}\text { AD 176, } \\
\text { Malan } 812\end{array}$ & Malvaceae & LP & 0.02 & 0.014 & Malaria & $\begin{array}{l}\text { Body smeared with mashed } \\
\text { leaves }\end{array}$ \\
\hline $\begin{array}{l}\text { Cola nitida (Vent.) } \\
\text { Schott \& Endl. }\end{array}$ & AD 163 & Malvaceae & LP & 0.02 & 0.009 & Whitlow Chickenpox & $\begin{array}{l}\text { Topical application with } \\
\text { crushed bark }\end{array}$ \\
\hline Costus afer Ker-Gawl. & Malan 1118 & Costaceae & $L P, K I$ & 0.02 & 0.008 & Palpitations & $\begin{array}{l}\text { Oral instillations with } \\
\text { crushed leaves }\end{array}$ \\
\hline $\begin{array}{l}\text { Dacryodes klaineana } \\
\text { (Pierre) Lam. }\end{array}$ & $\begin{array}{l}\text { Malan 777, } \\
898\end{array}$ & Burseraceae & $L P, K I$ & 0.02 & 0.007 & Dysmenorrhoea & $\begin{array}{l}\text { Enema with bark mashed } \\
\text { and diluted in water }\end{array}$ \\
\hline Daniellia thurifera Benn. & Malan 697 & Leguminosae & $L P, K I$ & 0.05 & 0.026 & Post-partum care & Bathing with leaves decoction \\
\hline Desmodium adscendens & Malan 443 & Leguminosae & $L P, K I$ & 0.05 & 0.04 & Pregnancy care & Enema with leaves mashed \\
\hline & & & & & & Diarrhoea & and diluted in water \\
\hline & & & & & & Wound & $\begin{array}{l}\text { Topical application with } \\
\text { crushed leaves }\end{array}$ \\
\hline
\end{tabular}


Table 1 List of medicinal plants used by Ehotile people (Continued)

\begin{tabular}{|c|c|c|c|c|c|c|c|}
\hline Digitaria horizontalis Willd. & Malan 874 & Poaceae & $L P, K I$ & 0.05 & 0.04 & Oedema & $\begin{array}{l}\text { Body smeared with mashed } \\
\text { whole plant }\end{array}$ \\
\hline Dioclea reflexa Hook.f. & Malan 994 & Leguminosae & $L P, K I$ & 0.02 & 0.009 & Infant fortification & $\begin{array}{l}\text { Entire seed soaked in infant } \\
\text { bathing water }\end{array}$ \\
\hline $\begin{array}{l}\text { Diospyros sanza-minika } \\
\text { A.Chev. }\end{array}$ & Malan 809 & Ebenaceae & $L P, K I$ & 0.02 & 0.003 & Giddiness & $\begin{array}{l}\text { Topical application with } \\
\text { crushed bark }\end{array}$ \\
\hline \multirow[t]{6}{*}{ Eclipta prostrata (L.) L. } & \multirow[t]{6}{*}{ Malan 752} & \multirow[t]{6}{*}{ Compositae } & \multirow[t]{6}{*}{$L P, K I$} & \multirow[t]{6}{*}{0.07} & \multirow[t]{6}{*}{0.041} & Hydrocephalus & $\begin{array}{l}\text { Body smeared with leaves } \\
\text { mashed }\end{array}$ \\
\hline & & & & & & Asema & Bathing with leaves decoction \\
\hline & & & & & & Giddiness & Eye drops with leaf juice \\
\hline & & & & & & Pregnancy care & \multirow{3}{*}{$\begin{array}{l}\text { Enema with leaves mashed } \\
\text { and diluted in water }\end{array}$} \\
\hline & & & & & & Malaria & \\
\hline & & & & & & Diarrhoea & \\
\hline Elaeis guineensis Jacq. & & Arecaceae & $L P, K I$ & 0.02 & 0.01 & Abortion & $\begin{array}{l}\text { Enema with mashed roots } \\
\text { diluted in water }\end{array}$ \\
\hline \multirow[t]{3}{*}{ Erythrina senegalensis DC. } & \multirow[t]{3}{*}{ Malan 856} & \multirow[t]{3}{*}{ Leguminosae } & \multirow[t]{3}{*}{ LP } & \multirow[t]{3}{*}{0.02} & \multirow[t]{3}{*}{0.006} & Rheumatism & $\begin{array}{l}\text { Topical application with } \\
\text { crushed bark }\end{array}$ \\
\hline & & & & & & Oedema & $\begin{array}{l}\text { Body smeared with } \\
\text { mashed leaves }\end{array}$ \\
\hline & & & & & & Dysmenorrhoea & $\begin{array}{l}\text { Enema with leaves mashed } \\
\text { and diluted in water }\end{array}$ \\
\hline $\begin{array}{l}\text { Euadenia trifoliolata } \\
\text { (Schumach. \& Thonn.) Oliv. }\end{array}$ & Malan 695 & Capparaceae & $L P, K I$ & 0.02 & 0.009 & Haemorrhoids & $\begin{array}{l}\text { Enema with mashed roots } \\
\text { diluted in water }\end{array}$ \\
\hline \multirow[t]{3}{*}{ Eugenia whytei Sprague } & \multirow[t]{3}{*}{ Malan 897} & \multirow[t]{3}{*}{ Myrtaceae } & \multirow[t]{3}{*}{$L P, K I$} & \multirow[t]{3}{*}{0.05} & \multirow[t]{3}{*}{0.021} & Bad breath & Roots as chewing stick \\
\hline & & & & & & Infant diarrhoea & $\begin{array}{l}\text { Enema with mashed leaves } \\
\text { diluted in water }\end{array}$ \\
\hline & & & & & & Nausea & Leaves chewed \\
\hline \multirow[t]{3}{*}{ Euphorbia hirta L. } & \multirow[t]{3}{*}{ AD 25} & \multirow[t]{3}{*}{ Euphorbiaceae } & \multirow[t]{3}{*}{$L P, K I$} & \multirow[t]{3}{*}{0.11} & \multirow[t]{3}{*}{0.048} & Measles & $\begin{array}{l}\text { Body smeared with mashed } \\
\text { leaves }\end{array}$ \\
\hline & & & & & & Pregnancy care & Enema with whole plant \\
\hline & & & & & & Diarrhoea & masnea and aliuted in water \\
\hline Ficus exasperata Vahl & AD 48 & Moraceae & LP & 0.02 & 0.009 & Injury & $\begin{array}{l}\text { Topical application with } \\
\text { crushed roots }\end{array}$ \\
\hline \multirow{2}{*}{$\begin{array}{l}\text { Flagellaria guineensis } \\
\text { Schumach. }\end{array}$} & \multirow[t]{2}{*}{ AD 04} & \multirow[t]{2}{*}{ Flagellariaceae } & \multirow[t]{2}{*}{$L P, K I$} & 0.04 & 0.005 & Malaria & Bathing with leaves decoction \\
\hline & & & & & & $\begin{array}{l}\text { Delay of motor } \\
\text { function }\end{array}$ & $\begin{array}{l}\text { Enema with roots mashed and } \\
\text { diluted in water }\end{array}$ \\
\hline Garcinia kola Heckel & AD 45 & Clusiaceae & LP & 0.04 & 0.025 & Bad breath & Roots as chewing stick \\
\hline & & & & & & Sexual asthenia & \\
\hline $\begin{array}{l}\text { Glyphaea brevis } \\
\text { (Spreng.) Monachino }\end{array}$ & AD 206 & Malvaceae & LP & 0.02 & 0.011 & Pregnancy care & $\begin{array}{l}\text { Enema with leaves mashed } \\
\text { and diluted in water }\end{array}$ \\
\hline Gossypium barbadense L. & AD 177 & Malvaceae & $L P, K I$ & 0.02 & 0.008 & Dysmenorrhoea & $\begin{array}{l}\text { Enema with leaves mashed } \\
\text { and diluted in water }\end{array}$ \\
\hline Hallea ledermannii & Malan 787, & Rubiaceae & $L P, K I$ & 0.04 & 0.013 & Malaria & Bathing with bark decoction \\
\hline & & & & & & Convulsion & $\begin{array}{l}\text { Body smeared with mashed } \\
\text { bark }\end{array}$ \\
\hline $\begin{array}{l}\text { Harungana madagascariensis } \\
\text { Lam. ex Poir. }\end{array}$ & Malan 719 & Hypericaceae & $L P, K I$ & 0.53 & 0.38 & Malaria & $\begin{array}{l}\text { Bark decoction drunk, taken as } \\
\text { enema and for bathing, Body } \\
\text { smeared with mashed bark }\end{array}$ \\
\hline $\begin{array}{l}\text { Heterotis rotundifolia } \\
\text { (Sm.) Jacq.-Fél. }\end{array}$ & Malan 552 & Melastomataceae & $L P, K I$ & 0.05 & 0.04 & Diarrhoea & $\begin{array}{l}\text { Enema with leaves mashed } \\
\text { and diluted in water }\end{array}$ \\
\hline Hoslundia opposita Vahl & Malan 953 & Lamiaceae & LP & 0.02 & 0.016 & Convulsion & Eye drops with leaf juice \\
\hline Icacina mannii Oliv. & Malan 1042 & Icacinaceae & $L P, K I$ & 0.15 & 0.083 & Dysmenorrhoea & Tuber decoction drunk \\
\hline
\end{tabular}


Table 1 List of medicinal plants used by Ehotile people (Continued)

\begin{tabular}{|c|c|c|c|c|c|c|c|}
\hline & & & & & & Sexual asthenia & \\
\hline & & & & & & Haemorrhoids & \\
\hline $\begin{array}{l}\text { Imperata cylindrica } \\
\text { (L.) Raeuschel }\end{array}$ & Malan 458 & Poaceae & $\mathrm{KI}$ & - & - & Convulsion & $\begin{array}{l}\text { Body smeared with mashed } \\
\text { leaves }\end{array}$ \\
\hline Jatropha curcas L. & AD 58 & Euphorbiaceae & LP & 0.02 & 0.009 & Abortion & $\begin{array}{l}\text { Enema with leaves mashed } \\
\text { and diluted in water }\end{array}$ \\
\hline Jatropha gossypiifolia L. & AD 139 & Euphorbiaceae & LP & 0.02 & 0.004 & Dysmenorrhoea & $\begin{array}{l}\text { Enema with leaves mashed } \\
\text { and diluted in water }\end{array}$ \\
\hline \multirow[t]{2}{*}{ Khaya ivorensis A.Chev. } & \multirow[t]{2}{*}{ Malan 419} & \multirow[t]{2}{*}{ Meliaceae } & \multirow[t]{2}{*}{ LP } & \multirow[t]{2}{*}{0.04} & \multirow[t]{2}{*}{0.019} & Haemorrhoids & $\begin{array}{l}\text { Enema with bark mashed } \\
\text { and diluted in water }\end{array}$ \\
\hline & & & & & & Sexual asthenia & $\begin{array}{l}\text { Bark soaked in local alcohol } \\
\text { and drunk }\end{array}$ \\
\hline \multirow[t]{2}{*}{$\begin{array}{l}\text { Kigelia africana } \\
\text { (Lam.) Benth. }\end{array}$} & \multirow[t]{2}{*}{ AD 201} & \multirow[t]{2}{*}{ Bignoniaceae } & \multirow[t]{2}{*}{$L P, K I$} & \multirow[t]{2}{*}{0.07} & \multirow[t]{2}{*}{0.048} & Swollen breasts & $\begin{array}{l}\text { Topical application with fruits, } \\
\text { leaves and bark crushed }\end{array}$ \\
\hline & & & & & & Sterility & $\begin{array}{l}\text { Enema with bark mashed and } \\
\text { diluted in water }\end{array}$ \\
\hline \multirow{2}{*}{$\begin{array}{l}\text { Lannea nigritana } \\
\text { (Scott-Elliot) Keay }\end{array}$} & \multirow[t]{2}{*}{ AD 95} & \multirow[t]{2}{*}{ Anacardiaceae } & \multirow[t]{2}{*}{ LP } & \multirow[t]{2}{*}{0.02} & \multirow[t]{2}{*}{0.006} & Ophtalmia & Eye drops with leaf juice \\
\hline & & & & & & Toohtache & $\begin{array}{l}\text { Mouthwash with leaves } \\
\text { decoction }\end{array}$ \\
\hline \multirow{3}{*}{$\begin{array}{l}\text { Lophira alata Banks } \\
\text { ex Gaertn. f. }\end{array}$} & \multirow[t]{3}{*}{ Malan 340} & \multirow[t]{3}{*}{ Ochnaceae } & \multirow[t]{3}{*}{ LP } & \multirow[t]{3}{*}{0.04} & \multirow[t]{3}{*}{0.012} & Bad breath & Roots as chewing stick \\
\hline & & & & & & Elokposan & Bathing with bark decoction \\
\hline & & & & & & Malaria & \\
\hline $\begin{array}{l}\text { Mallotus oppositifolius } \\
\text { (Geisel.) Müll.Arg. }\end{array}$ & \multirow[t]{7}{*}{ AD 278} & Euphorbiaceae & LP & 0.02 & 0.007 & Asema & Bathing with leaves decoction \\
\hline Mangifera indica $\mathrm{L}$. & & Anacardiaceae & $\mathrm{KI}$ & - & - & Malaria & Bathing with bark decoction \\
\hline \multirow[t]{5}{*}{ Manihot esculenta Crantz } & & \multirow[t]{5}{*}{ Euphorbiaceae } & \multirow[t]{5}{*}{$L P, K I$} & 0.09 & 0.022 & Headache & Nose drops with leaf juice \\
\hline & & & & & & Ophtalmia & Eye drops with leaf juice \\
\hline & & & & & & Infant diarrhoea & Enema with roasted fruits \\
\hline & & & & & & Diarrhoea & mashed and diluted in water \\
\hline & & & & & & Gonorrhoea & $\begin{array}{l}\text { Enema with leaves mashed } \\
\text { and diluted in water }\end{array}$ \\
\hline Manotes longiflora Baker & Malan 886 & Connaraceae & LP & 0.04 & 0.006 & Ophtalmia & Eye drops with leaf juice \\
\hline & & & & & & Dysenteria & $\begin{array}{l}\text { Enema with leaves mashed } \\
\text { and diluted in water }\end{array}$ \\
\hline $\begin{array}{l}\text { Mareya micrantha } \\
\text { (Benth.) Müll.Arg. }\end{array}$ & Malan 734 & Euphorbiaceae & $\mathrm{KI}$ & - & - & Childbirth & Leaves decoction drunk \\
\hline Microdesmis keayana & Malan 659 & Pandaceae & $L P, K I$ & 0.05 & 0.014 & Bad breath & Roots as chewing stick \\
\hline Leonard & & & & & & Dysmenorrhoea & $\begin{array}{l}\text { Enema with leaves mashed } \\
\text { and diluted in water }\end{array}$ \\
\hline $\begin{array}{l}\text { Microglossa pyrifolia } \\
\text { (Lam.) O.Ktze }\end{array}$ & AD 92 & Compositae & $L P, K I$ & 0.07 & 0.044 & Hydrocephalus & $\begin{array}{l}\text { Bathing with leaves decoction, } \\
\text { body smeared with mashed } \\
\text { leaves, leaves decoction drunk }\end{array}$ \\
\hline & & & & & & Asema & Bathing with leaves decoction \\
\hline & & & & & & Convulsion & $\begin{array}{l}\text { Nose drops with leaf juice, body } \\
\text { smeared with mashed leaves }\end{array}$ \\
\hline & & & & & & Pregnancy care & Enema with leaves mashed \\
\hline & & & & & & Dysmenorrhoea & diluted in water \\
\hline Microgramma owariensis & AD 39 & Polypodiaceae & $L P, K I$ & 0.05 & 0.028 & Sterility & Enema with whole plant \\
\hline (Desv.) $A$ & & & & & & Dysmenorrhoea & ted in water \\
\hline $\begin{array}{l}\text { Microsorum scolopendria } \\
\text { (Burm.f.) Copel. }\end{array}$ & AD 57 & Polypodiaceae & $L P, K I$ & 0.04 & 0.014 & Dysmenorrhoea & $\begin{array}{l}\text { Enema with whole plant } \\
\text { mashed and diluted in water }\end{array}$ \\
\hline
\end{tabular}


Table 1 List of medicinal plants used by Ehotile people (Continued)

\begin{tabular}{|c|c|c|c|c|c|c|c|}
\hline \multirow[t]{3}{*}{$\begin{array}{l}\text { Milicia excelsa (Welw.) } \\
\text { C.C.Berg }\end{array}$} & \multirow[t]{3}{*}{ Malan 785} & \multirow[t]{3}{*}{ Moraceae } & \multirow[t]{3}{*}{$\mathrm{KI}$} & \multirow[t]{3}{*}{-} & \multirow[t]{3}{*}{-} & Hydrocephalus & $\begin{array}{l}\text { Bathing with bark decoction, } \\
\text { body smeared with mashed } \\
\text { bark }\end{array}$ \\
\hline & & & & & & Dysenteria & \multirow{2}{*}{$\begin{array}{l}\text { Enema with mashed bark } \\
\text { diluted in water }\end{array}$} \\
\hline & & & & & & Butumanklan & \\
\hline \multirow[t]{2}{*}{ Mitracarpus scaber Zucc. } & \multirow[t]{2}{*}{ AD 156} & \multirow[t]{2}{*}{ Rubiaceae } & \multirow[t]{2}{*}{ LP } & \multirow[t]{2}{*}{0.01} & \multirow[t]{2}{*}{0.018} & Dry patchs & $\begin{array}{l}\text { Topical application with } \\
\text { crushed leaves }\end{array}$ \\
\hline & & & & & & Lumbar pain & $\begin{array}{l}\text { Enema with mashed leaves } \\
\text { diluted in water }\end{array}$ \\
\hline \multirow[t]{3}{*}{ Momordica charantia L. } & \multirow[t]{3}{*}{ Malan 1011} & \multirow[t]{3}{*}{ Cucurbitaceae } & \multirow[t]{3}{*}{$L P, K I$} & \multirow[t]{3}{*}{0.11} & \multirow[t]{3}{*}{0.029} & Malaria & \multirow{2}{*}{$\begin{array}{l}\text { Bathing with leaves decoction, } \\
\text { body smeared with mashed } \\
\text { leaves }\end{array}$} \\
\hline & & & & & & Chickenpox & \\
\hline & & & & & & Abortion & $\begin{array}{l}\text { Body smeared with mashed } \\
\text { leaves, Enema with seeds } \\
\text { mashed and diluted in water }\end{array}$ \\
\hline $\begin{array}{l}\text { Monodora myristica } \\
\text { (Gaertn.) Dunal }\end{array}$ & Malan 780 & Annonaceae & $L P, K I$ & 0.01 & 0.029 & Haemorrhoids & $\begin{array}{l}\text { Seeds added to enema } \\
\text { preparation }\end{array}$ \\
\hline Morinda lucida G.Don & AD 175 & Rubiaceae & $\mathrm{KI}$ & - & - & Infant diarrhoea & $\begin{array}{l}\text { Enema with bark mashed and } \\
\text { diluted in water }\end{array}$ \\
\hline \multirow[t]{2}{*}{ Musa paradisiaca L. } & & \multirow[t]{2}{*}{ Musaceae } & \multirow[t]{2}{*}{$\mathrm{Kl}$} & \multirow[t]{2}{*}{-} & \multirow[t]{2}{*}{-} & Malaria & Bathing with leaves decoction \\
\hline & & & & & & Ophtalmia & Eye drops with sap \\
\hline \multirow[t]{2}{*}{$\begin{array}{l}\text { Nephrolepis biserrata } \\
\text { (Sw.) Schott }\end{array}$} & \multirow[t]{2}{*}{ Malan 599} & Nephrolepidaceae & $L P, K I$ & 0.05 & 0.009 & Remove splinters & $\begin{array}{l}\text { Topical application with } \\
\text { crushed leaves }\end{array}$ \\
\hline & & & & & & Dysmenorrhoea & $\begin{array}{l}\text { Enema with leaves mashed } \\
\text { and diluted in water }\end{array}$ \\
\hline $\begin{array}{l}\text { Newbouldia laevis } \\
\text { (P. Beauv.) Seem. }\end{array}$ & Malan 1027 & Bignoniaceae & $L P, K I$ & 0.05 & 0.024 & Dysmenorrhoea & $\begin{array}{l}\text { Enema with leaves mashed } \\
\text { and diluted in water }\end{array}$ \\
\hline ex Bureau & & & & & & Hoarseness & Bark decoction drunk \\
\hline & & & & & & Raging toothache & $\begin{array}{l}\text { Mouthwash with bark } \\
\text { decoction }\end{array}$ \\
\hline & & & & & & Dysmenorrhoea & $\begin{array}{l}\text { Enema with mashed bark } \\
\text { diluted in water }\end{array}$ \\
\hline & & & & & & Remove splinters & $\begin{array}{l}\text { Topical application with } \\
\text { crushed leaves }\end{array}$ \\
\hline & & & & & & Fracture & $\begin{array}{l}\text { Topical application with } \\
\text { crushed bark }\end{array}$ \\
\hline Nymphaea lotus L. & Malan 925 & Nympheaceae & LP & 0.04 & 0.008 & Pregnancy care & Enema with leaves mashed \\
\hline & & & & & & $\begin{array}{l}\text { Delay of motor } \\
\text { function }\end{array}$ & dna almuted in water \\
\hline $\begin{array}{l}\text { Ocimum americanum } \\
\text { var. americanum } \mathrm{L} \text {. }\end{array}$ & AD 16 & Lamiaceae & $L P, K I$ & 0.04 & 0.014 & Lumbar pain & $\begin{array}{l}\text { Enema with leaves mashed } \\
\text { and diluted in water }\end{array}$ \\
\hline & & & & & & Swollen breasts & $\begin{array}{l}\text { Topical application with } \\
\text { crushed leaves }\end{array}$ \\
\hline & & & & & & Convulsion & Eye drops with leaf juice \\
\hline Ocimum gratissimum L. & Malan 1009 & Lamiaceae & $L P, K I$ & 0.38 & 0.245 & Convulsion & Eye drops with leaf juice \\
\hline & & & & & & Headache & Nose drops with leaf juice \\
\hline & & & & & & Cough & $\begin{array}{l}\text { Nose or eye drops with } \\
\text { leaf juice }\end{array}$ \\
\hline & & & & & & Fever attack & Enema with mashed leaves \\
\hline & & & & & & Malaria & 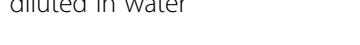 \\
\hline & & & & & & Dysmenorrhoea & \\
\hline & & & & & & Pregnancy care & \\
\hline
\end{tabular}


Table 1 List of medicinal plants used by Ehotile people (Continued)

\begin{tabular}{|c|c|c|c|c|c|c|c|}
\hline & & & & & & Infant diarrhoea & \\
\hline & & & & & & Stomachache & \\
\hline \multirow[t]{2}{*}{$\begin{array}{l}\text { Palisota hirsuta } \\
\text { (Thunb.) Engl. }\end{array}$} & \multirow[t]{2}{*}{ Malan 984} & \multirow[t]{2}{*}{ Commelinaceae } & \multirow[t]{2}{*}{$\mathrm{KI}$} & \multirow[t]{2}{*}{-} & \multirow[t]{2}{*}{-} & $\begin{array}{l}\text { Delay of motor } \\
\text { function }\end{array}$ & \multirow[t]{2}{*}{$\begin{array}{l}\text { Enema with leaves mashed } \\
\text { and diluted in water }\end{array}$} \\
\hline & & & & & & Dysmenorrhoea & \\
\hline \multirow[t]{3}{*}{ Parinari excelsa Sabine } & \multirow[t]{3}{*}{ AD 148} & \multirow[t]{3}{*}{ Chrysobalanaceae } & \multirow[t]{3}{*}{$\mathrm{Kl}$} & \multirow[t]{3}{*}{-} & \multirow[t]{3}{*}{-} & Sexual asthenia & $\begin{array}{l}\text { Bark soaked in local alcohol } \\
\text { and drunk }\end{array}$ \\
\hline & & & & & & Haemorrhoids & \multirow{2}{*}{$\begin{array}{l}\text { Enema with bark mashed } \\
\text { and diluted in water }\end{array}$} \\
\hline & & & & & & Dysmenorrhoea & \\
\hline Paullinia pinnata $\mathrm{L}$. & $\begin{array}{l}\text { AD 124, } \\
\text { Malan } 853\end{array}$ & Sapindaceae & $L P, K I$ & 0.2 & 0.122 & Hydrocephalus & Bathing with leaves decoction \\
\hline \multirow[t]{2}{*}{$\begin{array}{l}\text { Pentaclethra } \\
\text { macrophylla Benth. }\end{array}$} & \multirow[t]{2}{*}{ Malan 1110} & \multirow[t]{2}{*}{ Leguminosae } & \multirow[t]{2}{*}{$L P, K I$} & \multirow[t]{2}{*}{0.05} & \multirow[t]{2}{*}{0.03} & Hydrocephalus & $\begin{array}{l}\text { Bathing with bark decoction, } \\
\text { body smeared with mashed } \\
\text { bark }\end{array}$ \\
\hline & & & & & & Malaria & $\begin{array}{l}\text { Body smeared with mashed } \\
\text { bark }\end{array}$ \\
\hline $\begin{array}{l}\text { Phaulopsis ciliata } \\
\text { (Willd.) Hepper }\end{array}$ & Malan 954 & Acanthaceae & $\mathrm{Kl}$ & - & - & Colic & $\begin{array}{l}\text { Enema with leaves mashed } \\
\text { and diluted in water }\end{array}$ \\
\hline \multirow[t]{3}{*}{$\begin{array}{l}\text { Phyllanthus amarus } \\
\text { Schumach. \& Thonn. }\end{array}$} & \multirow[t]{3}{*}{ AD 144} & \multirow[t]{3}{*}{ Phyllanthaceae } & \multirow[t]{3}{*}{$L P, K I$} & \multirow[t]{3}{*}{0.13} & \multirow[t]{3}{*}{0.037} & Chickenpox & $\begin{array}{l}\text { Body smeared with whole } \\
\text { plant mashed }\end{array}$ \\
\hline & & & & & & Pregnancy care & $\begin{array}{l}\text { Enema with leaves mashed } \\
\text { and diluted in water }\end{array}$ \\
\hline & & & & & & Malaria & Whole plant decoction drunk \\
\hline $\begin{array}{l}\text { Picralima nitida } \\
\text { (Stapf) T. \& H.Durand }\end{array}$ & AD 237 & Apocynaceae & $L P, K I$ & 0.09 & 0.082 & Inguinal hernia & $\begin{array}{l}\text { Enema with seeds mashed } \\
\text { and diluted in water }\end{array}$ \\
\hline $\begin{array}{l}\text { Piptadeniastrum } \\
\text { africanum (Hook.) Brenan }\end{array}$ & Malan 1108 & Leguminosae & $\mathrm{KI}$ & - & - & Inguinal hernia & $\begin{array}{l}\text { Enema with bark mashed } \\
\text { and diluted in water }\end{array}$ \\
\hline \multirow[t]{2}{*}{ Pleioceras barteri Baill. } & \multirow[t]{2}{*}{ Malan 700} & \multirow[t]{2}{*}{ Apocynaceae } & \multirow[t]{2}{*}{$\mathrm{Kl}$} & - & - & Boil & Topical application \\
\hline & & & & & & Oedema & \\
\hline Plumbago zeylanica L. & Malan 873 & Plumbaginaceae & $L P, K I$ & 0.04 & 0.004 & Inguinal hernia & $\begin{array}{l}\text { Enema with leaves and roots } \\
\text { mashed and diluted in water }\end{array}$ \\
\hline Psidium guajava $\mathrm{L}$. & & Myrtaceae & $\mathrm{Kl}$ & - & - & Butumanklan & $\begin{array}{l}\text { Enema with roots mashed } \\
\text { and diluted water }\end{array}$ \\
\hline $\begin{array}{l}\text { Pterocarpus } \\
\text { santalinoides DC. }\end{array}$ & $\begin{array}{l}\text { AD 09, AD } \\
59\end{array}$ & Leguminosae & $\mathrm{KI}$ & - & - & Malaria & $\begin{array}{l}\text { Body smeared with mashed } \\
\text { bark }\end{array}$ \\
\hline $\begin{array}{l}\text { Pycnanthus } \\
\text { angolensis }\end{array}$ & AD 212 & Myristicaceae & $L P, K I$ & 0.05 & 0.022 & Haemorrhoids & $\begin{array}{l}\text { Enema with bark mashed } \\
\text { and diluted in water }\end{array}$ \\
\hline (Welw.) Warb. & & & & & & Anaemia & Bark decoction drunk \\
\hline $\begin{array}{l}\text { Rauvolfia vomitoria } \\
\text { Afzel. }\end{array}$ & AD 36 & Apocynaceae & $L P, K I$ & 0.07 & 0.018 & Malaria & $\begin{array}{l}\text { Body smeared with mashed } \\
\text { roots, enema with roots } \\
\text { mashed and diluted in water }\end{array}$ \\
\hline & & & & & & Sexual asthenia & $\begin{array}{l}\text { Roots soaked in local alcohol } \\
\text { and drunk }\end{array}$ \\
\hline & & & & & & Inguinal hernia & $\begin{array}{l}\text { Enema with mashed roots } \\
\text { diluted in water }\end{array}$ \\
\hline & & & & & & Rheumatism & $\begin{array}{l}\text { Topical application with } \\
\text { crushed bark }\end{array}$ \\
\hline $\begin{array}{l}\text { Rhizophora } \\
\text { racemosa G.F.W.Mey. }\end{array}$ & Malan 806 & Rhizophoraceae & $\mathrm{Kl}$ & - & - & Hydrocephalus & $\begin{array}{l}\text { Bathing with bark decoction } \\
\text { and body smeared with } \\
\text { mashed bark }\end{array}$ \\
\hline Ricinodendron & Malan 669 & Euphorbiaceae & LP & 0.04 & 0.004 & Dysmenorrhoea & Enema with bark mashed \\
\hline $\begin{array}{l}\text { heudelotii (Baill.) } \\
\text { Pierre ex Heckel }\end{array}$ & & & & & & Haemorrhoids & \\
\hline
\end{tabular}


Table 1 List of medicinal plants used by Ehotile people (Continued)

\begin{tabular}{|c|c|c|c|c|c|c|c|}
\hline \multirow[t]{2}{*}{ Ricinus communis L. } & & Euphorbiaceae & $\mathrm{KI}$ & - & - & Headache & $\begin{array}{l}\text { Topical application with } \\
\text { mashed bark }\end{array}$ \\
\hline & & & & & & Lumbar pain & Massage with warm leaves \\
\hline Saccharum officinarum L. & & Poaceae & $\mathrm{KI}$ & - & - & Abortion & $\begin{array}{l}\text { Enema with leaves mashed } \\
\text { and diluted in water }\end{array}$ \\
\hline \multirow[t]{2}{*}{$\begin{array}{l}\text { Secamone afzelii } \\
\text { (Schultes) K.Schum. }\end{array}$} & AD 375 & Apocynaceae & $\mathrm{KI}$ & - & - & Lumbar pain & $\begin{array}{l}\text { Enema with leaves mashed } \\
\text { and diluted in water }\end{array}$ \\
\hline & & & & & & Asthma & Leaves decoction drunk \\
\hline $\begin{array}{l}\text { Smeathmannia pubescens } \\
\text { Soland. ex R.Br. }\end{array}$ & $\begin{array}{l}\text { AD 171, } \\
\text { Malan } 863\end{array}$ & Passifloraceae & $\mathrm{KI}$ & - & - & Bad breath & Roots as chewing stick \\
\hline \multirow[t]{3}{*}{ Solanum lycopersicum L. } & & Solanaceae & $L P, K I$ & 0.07 & 0.018 & $\begin{array}{l}\text { Serious disease of } \\
\text { unknown origin }\end{array}$ & Eye drops with leaf juice \\
\hline & & & & & & Injury & $\begin{array}{l}\text { Topical application with } \\
\text { crushed leaves }\end{array}$ \\
\hline & & & & & & Contraception & $\begin{array}{l}\text { Enema with leaves mashed } \\
\text { and diluted in water }\end{array}$ \\
\hline Solanum nigrum L. & Malan 1022 & Solanaceae & LP & 0.04 & 0.002 & Malaria & $\begin{array}{l}\text { Enema with leaves mashed } \\
\text { and diluted in water }\end{array}$ \\
\hline \multirow{3}{*}{$\begin{array}{l}\text { Solenostemon } \\
\text { monostachyus } \\
\text { (P.Beauv.) Briq }\end{array}$} & \multirow[t]{3}{*}{ Malan 982} & \multirow[t]{3}{*}{ Lamiaceae } & \multirow[t]{3}{*}{$\mathrm{Kl}$} & \multirow[t]{3}{*}{-} & \multirow[t]{3}{*}{-} & Laryngitis & Leaf juice swallowed \\
\hline & & & & & & Haedache & \multirow[t]{2}{*}{ Nose drops with leaf juice } \\
\hline & & & & & & Mental illness & \\
\hline $\begin{array}{l}\text { Spathodea } \\
\text { campanulata P.Beauv. }\end{array}$ & AD 116 & Bignoniaceae & LP & 0.05 & 0.035 & Malaria & $\begin{array}{l}\text { Bark decoction drunk, taken } \\
\text { as enema and for bathing, } \\
\text { body smeared with mashed } \\
\text { bark }\end{array}$ \\
\hline \multirow[t]{2}{*}{ Spondias mombin L. } & \multirow[t]{2}{*}{ AD 217} & \multirow[t]{2}{*}{ Anacardiaceae } & \multirow[t]{2}{*}{ LP } & \multirow[t]{2}{*}{0.04} & \multirow[t]{2}{*}{0.017} & Diarrhoea & \multirow{2}{*}{$\begin{array}{l}\text { Enema with leaves mashed } \\
\text { and diluted in water }\end{array}$} \\
\hline & & & & & & Post-partum care & \\
\hline $\begin{array}{l}\text { Strombosia pustulata } \\
\text { var. lucida (Léonard) } \\
\text { Villiers }\end{array}$ & Malan 816 & Olacaceae & $\mathrm{Kl}$ & - & - & Bad breath & Roots as chewing stick \\
\hline \multirow{3}{*}{$\begin{array}{l}\text { Syzygium guineense } \\
\text { var. littorale Keay }\end{array}$} & \multirow{3}{*}{$\begin{array}{l}\text { AD 140, AD } \\
158\end{array}$} & \multirow[t]{3}{*}{ Myrtaceae } & \multirow[t]{3}{*}{ LP } & \multirow[t]{3}{*}{0.05} & \multirow[t]{3}{*}{0.011} & Butumanklan & \multirow{3}{*}{$\begin{array}{l}\text { Enema with bark mashed } \\
\text { and diluted in water }\end{array}$} \\
\hline & & & & & & Diarrhoea & \\
\hline & & & & & & Stomachache & \\
\hline Tetracera alnifolia Willd. & AD 232 & Dilleniaceae & $\mathrm{KI}$ & - & - & Inner ear cleanning & Ear drops with sap \\
\hline $\begin{array}{l}\text { Tetrapleura tetraptera } \\
\text { (Schumach. \& Thonn.) } \\
\text { Taub. }\end{array}$ & AD 143 & Leguminosae & $\mathrm{KI}$ & - & - & Impotence & $\begin{array}{l}\text { Enema with bark mashed } \\
\text { and diluted in water }\end{array}$ \\
\hline Tiliacora dinklagei Engl. & Malan 884 & Menispermaceae & $\mathrm{Kl}$ & - & - & Sexual asthenia & $\begin{array}{l}\text { Roots soaked in local alcohol } \\
\text { and drunk }\end{array}$ \\
\hline \multirow{2}{*}{$\begin{array}{l}\text { Trema guineensis } \\
\text { Schumach. \& Thonn. }\end{array}$} & \multirow[t]{2}{*}{ Malan 676} & \multirow[t]{2}{*}{ Cannabaceae } & \multirow[t]{2}{*}{ LP } & \multirow[t]{2}{*}{0.04} & \multirow[t]{2}{*}{0.005} & Stomachache & Enema with leaves mashed \\
\hline & & & & & & Pregnancy care & 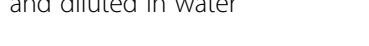 \\
\hline Uvaria afzelii Scott-Elliot & $\begin{array}{l}\text { Malan 793, } \\
\text { AD } 62\end{array}$ & Annonaceae & $L P, K I$ & 0.22 & 0.123 & Hydrocephalus & $\begin{array}{l}\text { Bathing with decoction of } \\
\text { roots and leaves, body smeared } \\
\text { with leaves and roots mashed }\end{array}$ \\
\hline & & & & & & Pregnancy care & $\begin{array}{l}\text { Enema with leaves mashed } \\
\text { and diluted in water }\end{array}$ \\
\hline & & & & & & Colic & Decoction of leaves drunk \\
\hline Uvaria chamae P.Beauv. & $\begin{array}{l}\text { Malan } 703 \\
\text { AD } 106\end{array}$ & Annonaceae & $L P, K I$ & 0.09 & 0.068 & Diaper dermatitis & $\begin{array}{l}\text { Body smeared with roots } \\
\text { mashed }\end{array}$ \\
\hline & & & & & & Measles & $\begin{array}{l}\text { Topical application with } \\
\text { crushed roots and leaves }\end{array}$ \\
\hline & & & & & & Chickenpox & $\begin{array}{l}\text { Body smeared with mashed } \\
\text { roots }\end{array}$ \\
\hline
\end{tabular}


Table 1 List of medicinal plants used by Ehotile people (Continued)

\begin{tabular}{|c|c|c|c|c|c|c|c|}
\hline \multirow{2}{*}{$\begin{array}{l}\text { Uvariastrum insculptum } \\
\text { (Engl. \& Diels) } \\
\text { Spargue \& Hutch. }\end{array}$} & \multirow[t]{2}{*}{ Malan 912} & \multirow[t]{2}{*}{ Annonaceae } & \multirow[t]{2}{*}{$\mathrm{Kl}$} & \multirow[t]{2}{*}{-} & \multirow[t]{2}{*}{-} & Bad breath & Roots as chewing stick \\
\hline & & & & & & Sexual asthenia & $\begin{array}{l}\text { Roots soaked in local alcohol } \\
\text { and drunk }\end{array}$ \\
\hline \multirow{4}{*}{$\begin{array}{l}\text { Vernonia amygdalina } \\
\text { Delile }\end{array}$} & \multirow[t]{4}{*}{ AD 131} & \multirow[t]{4}{*}{ Compositae } & \multirow[t]{4}{*}{$L P, K I$} & \multirow[t]{4}{*}{0.11} & \multirow[t]{4}{*}{0.051} & Diarrhoea & \multirow{2}{*}{$\begin{array}{l}\text { Enema with leaves mashed } \\
\text { and diluted in water }\end{array}$} \\
\hline & & & & & & Helminthiasis & \\
\hline & & & & & & Malaria & \multirow{2}{*}{$\begin{array}{l}\text { Bathing with leaves decoction, } \\
\text { body smeared with mashed } \\
\text { leaves }\end{array}$} \\
\hline & & & & & & Chickenpox & \\
\hline Vernonia conferta Benth. & AD 187 & Compositae & $L P, K I$ & 0.07 & 0.059 & Malaria & Leaves decoction drunk \\
\hline Vitex oxycuspis Baker & AD 234 & Lamiaceae & $\mathrm{Kl}$ & - & - & Haemorrhoids & $\begin{array}{l}\text { Enema with bark mashed } \\
\text { and diluted in water }\end{array}$ \\
\hline \multirow{2}{*}{$\begin{array}{l}\text { Xylopia acutiflora } \\
\text { (Dunal) A.Rich. }\end{array}$} & \multirow[t]{2}{*}{ Malan 916} & \multirow[t]{2}{*}{ Annonaceae } & \multirow[t]{2}{*}{ LP } & \multirow[t]{2}{*}{0.04} & \multirow[t]{2}{*}{0.016} & Bad breath & Roots as chewing stick \\
\hline & & & & & & Sexual asthenia & $\begin{array}{l}\text { Roots soaked in local alcohol } \\
\text { and drunk }\end{array}$ \\
\hline $\begin{array}{l}\text { Xylopia aethiopica } \\
\text { (Dunal) A.Rich. }\end{array}$ & Malan 711 & Annonaceae & $L P, K I$ & 0.29 & 0.225 & Post-partum care & $\begin{array}{l}\text { Enema with fruits mashed } \\
\text { and diluted in water }\end{array}$ \\
\hline \multirow[t]{8}{*}{$\begin{array}{l}\text { Zanthoxylum gilletii } \\
\text { (De Wild.) Waterman }\end{array}$} & \multirow[t]{8}{*}{ Malan 742} & \multirow[t]{8}{*}{ Rutaceae } & \multirow[t]{8}{*}{$\mathrm{LP}, \mathrm{Kl}$} & \multirow[t]{8}{*}{0} & \multirow[t]{8}{*}{0.111} & Fever attack & $\begin{array}{l}\text { Body smeared with mashed } \\
\text { bark }\end{array}$ \\
\hline & & & & & & Sexual asthenia & $\begin{array}{l}\text { Bark soaked in local alcohol } \\
\text { and drunk }\end{array}$ \\
\hline & & & & & & Laryngitis & Bark decoction drunk \\
\hline & & & & & & Malaria & $\begin{array}{l}\text { Body smeared with mashed } \\
\text { bark }\end{array}$ \\
\hline & & & & & & Cough & Bark decoction drunk \\
\hline & & & & & & Colic & \multirow{3}{*}{$\begin{array}{l}\text { Enema with bark mashed } \\
\text { and diluted in water }\end{array}$} \\
\hline & & & & & & Haemorrhoids & \\
\hline & & & & & & Post-partum care & \\
\hline
\end{tabular}

Frequency of citation (Fq) and Smith' index (Smith' S), only for the survey with laypeople (LP). Kl: key informant.

\section{(\%)}

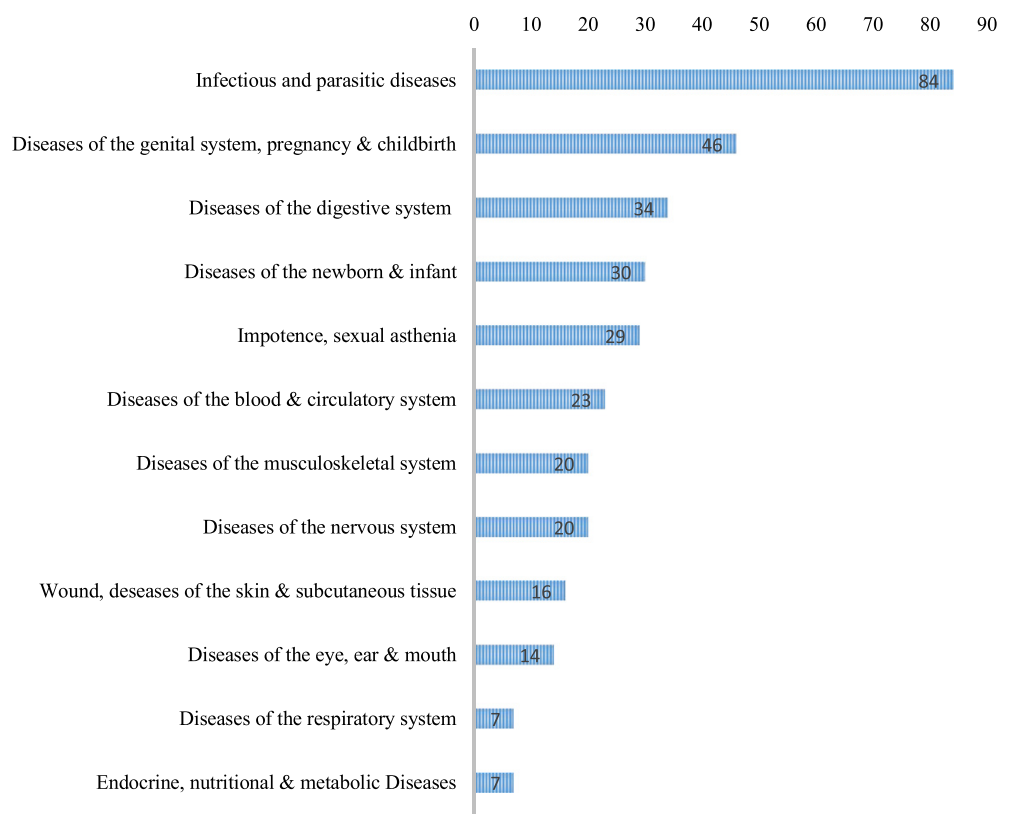

Figure 3 Diseases categories in relation to medicinal plants used by ehotile people. 
Waterman $(\mathrm{Fq}=0.63)$ and Microdesmis keayana $\mathrm{J}$. Léonard $(\mathrm{Fq}=0.63)$.

ICF ranges from 0 to 0.42 , implying that there is no real consensus in the medicinal use of plants (Table 2). However, the highest values were recorded for infectious \& parasitic diseases $(\mathrm{ICF}=0.42)$, diseases of the blood \& circulatory system $(\mathrm{ICF}=0.30)$. The lowest ICF were recorded for diseases of the eye, ear $(\mathrm{ICF}=0) \&$ mouth and diseases of the respiratory system $(\mathrm{ICF}=0)$. In the mental and behavioural disorders, only one indication was given for a single plant: Solenostemon monostachyus (P.Beauv.) Briq., used to treat or calm people with mental disorders.

The question of the availability of the resources was discussed with our experts. According to these specialists, excluded the exotic or domesticated plants (14.7\%), 4 types for the $85.3 \%$ of native medicinal plants can be distinguished.

- Abundant plants, with organs sought-after easy to collect (44.6\%), such as Ageratum conyzoides L., Alchornea cordifolia (Schumach. \& Thonn.) Müll. Arg. or Baphia nitida Lodd.

- Abundant plants, but organs sought-after difficult to harvest or rare (10.2\%) such as Tetracera alnifolia Willd. or Kigelia africana (Lam.) Benth.

- Scarce plants in the region (24.7\%) such as Alstonia boonei, Momordica charantia L., Zanthoxylum gilletii (Figure 4).

- Endangered plants in the region, rarely found in their natural habitat (5.8\%) such as Garcinia kola Heckel, Khaya ivorensis A.Chev. or Picralima nitida (Stapf) T.Durand \& H.Durand.

Table $\mathbf{2}$ Key informant consensus by diseases category

\begin{tabular}{llll}
\hline Category & Species & Citation & ICF \\
\hline Infectious \& parasitic diseases & 31 & 53 & 0.42 \\
$\begin{array}{l}\text { Diseases of the blood \& circulatory } \\
\text { system }\end{array}$ & 20 & 28 & 0.30 \\
$\begin{array}{l}\text { Wound, diseases of the skin \& } \\
\text { subcutaneous tissue }\end{array}$ & 14 & 19 & 0.28 \\
$\begin{array}{l}\text { Diseases of the genital system, } \\
\text { pregnancy \& childbirth }\end{array}$ & 23 & 31 & 0.27 \\
$\begin{array}{l}\text { Diseases of the newborn \& infant } \\
\text { Diseases of the digestive system }\end{array}$ & 15 & 20 & 0.26 \\
$\begin{array}{l}\text { Impotence \& sexual asthenia } \\
\text { Diseases of the musculoskeletal system }\end{array}$ & 11 & 17 & 0.19 \\
Diseases of the eye, ear \& mouth & 12 & 9 & 0.13 \\
Diseases of the respiratory system & 5 & 5 & 0.09 \\
Mental \& behavioural disorders & 1 & 12 & 0.00 \\
\hline
\end{tabular}

ICF: informant consensus factor.

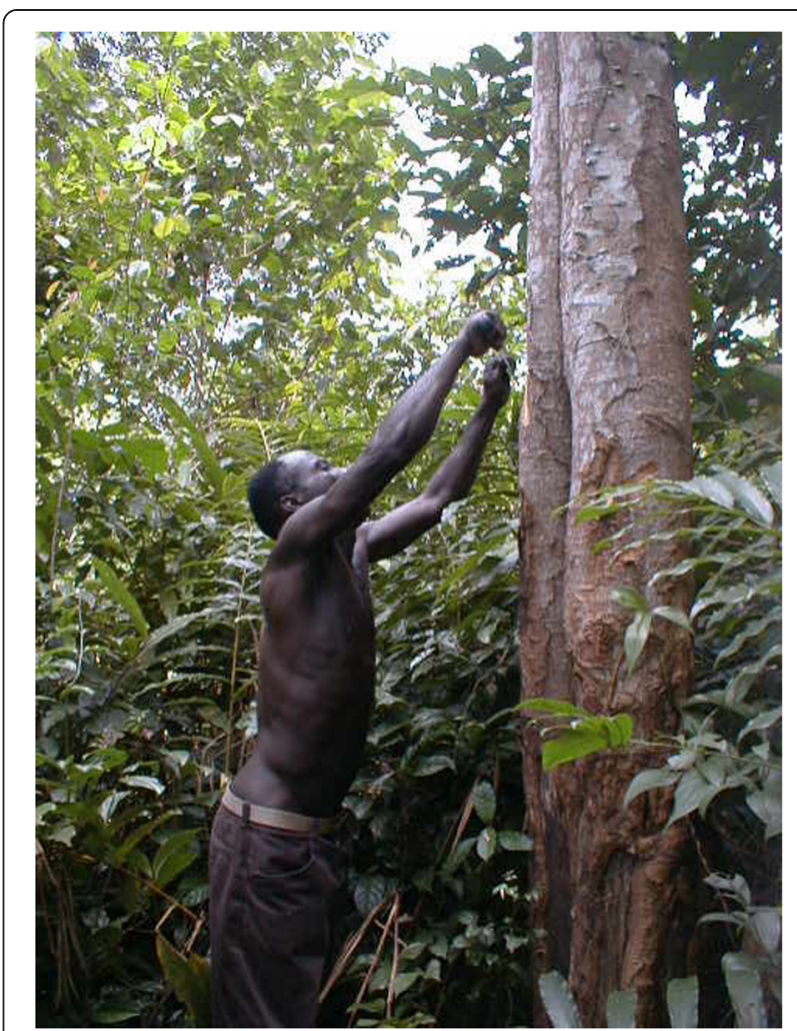

Figure 4 Harvesting stem bark of zanthoxylum gilletii one the ehotile major medicinal plants. This tree is becoming scarce in ehotile land and the stem bark difficult to harvest. Note the numerous torn stems at the base of the tree, due to frequent harvest.

According to this information, $59.3 \%$ of medicinal plants used were easily accessible (44.6\% of native abundant species and 14.7 of exotic domesticated or cultivated species), while $5.8 \%$ were hardly ever found in the region. About the categories of diseases, nearly $80 \%$ of plants used in the treatment of wounds and skin infections were easy to find, while $15 \%$ of species used as sexual stimulants (Garcinia kola and Khaya ivorensis) were very rare in the study area.

Concerning the used parts (Table 3), in the category of "abundant plants, with organs sought-after easy to collect", leaves constituted $53.6 \%$ of parts used, while the roots were highly sought $(50 \%)$ in the category of "endangered plants in the region, rarely found in their natural habitat".

\section{Discussion}

Most of ethnobotanical studies in West Africa are clearly marked as concerning the use of plants as medicine or as food [7]. Regarding medicinal uses, several works have been carried out about the treatment of several microbial infections [32,33], a range of tropical endemic 
Table 3 Proportion of medicinal plants part used in relation to plants availability type

\begin{tabular}{lllllll}
\hline Availability type & \multicolumn{3}{l}{ Proportion (\%) of parts used } \\
\cline { 2 - 6 } & Leaf & Bark & Root \& tuber & Fruit \& seed & Exudate & Whole plant \\
\hline Abundant plants \& part used easy to collect & 53.6 & 16.1 & 19.6 & 1.8 & 1.8 & 7.1 \\
Abundant plants. but part used difficult to harvest or rare & 20.0 & 40.0 & 13.3 & 13.3 & 6.7 & 6.7 \\
Scarce plants in the region & 19.0 & 61.9 & 14.3 & 4.8 & 0.0 & 0.0 \\
Endangered plants in the region. rarely found in their natural habitat & 0.0 & 25.0 & 50.0 & 25.0 & 0.0 & 0.0 \\
Exotic domesticated or cultivated plants & 77.8 & 14.8 & 7.4 & 0.0 & 0.0 & 0.0 \\
\hline
\end{tabular}

diseases including malaria [34-38] as well as diabetes $[39,40]$ and diverse infections [41].

This propensity of African ethnobotanists to medicinal plants reflects the fact that the study of plants used traditionally as medicines is an interesting discipline because of the possibility to find new drugs [42]. This has prompted many research teams to carry out studies on plants used in Africa by traditional healers against diseases.

Furthermore, these studies have the laudable aim of creating databases on traditional knowledge of plants use, although they remain disparate. Nevertheless, this abundant literature shows that despite the advances in Western medicine, African traditional medicine has gained renewed interest in the health care services throughout the continent. This could probably be due to the rapidly increasing awareness of the potential and curative abilities of alternative medicines, especially from the use of medicinal plants [42], as well as the inadequate access to Western medicine and physicians and the high cost for Western drugs [3].

This is the situation around the Aby Lagoon. Indeed, the results of this study showed that Ehotile people use plants in traditional medicinal practices. Globally, they know and use the handy plants as first treatment of common ailments. However, they also often resort to healer's services. These healers are more or less known for specific field including reproductive health, rheumatism, and mental health. This specialization can justify the low values of ICF $(<0.5)$. Indications of some plants, such as Harungana madagascariensis, Ocimum gratissimum, are widely shared, but information on plants and practices related to diseases specifically treated by healers is well protected. Thus, the recourse to these specialists is generally based on their area of recognized competence.

Though, as noted, the main purpose of these recourses remains what we called "African-disorders": Asema, elokposan or butumanklan. Asema, for example, is a child disease manifested by an under nutrition. This condition would be due to "impurities" related to the resumption of sexual activity of the mother before her child is strengthened. This fact elicits a physical degradation of the child followed by frequent diarrhoea and signs of emotional deprivation. This disease is well known in sub-Saharan Africa. Such signs, related to the same cause, have been described in the Baka pygmies in Cameroon [43] and the Democratic Republic of Congo [44]. So these authors classified them as "African diseases" maintaining the local name (Buse in DRC) or assigning an approximate equivalence ("child-cross" in Cameroon).

According to the description of the signs, the elokposan would be the Pott's disease that is a bone location of tuberculosis inducing developments of a hump. However, according to popular belief, elokposan is an occult disease. Thus, to protect themselves against it, wooden statues, also called elokposan, representing hunchbacks were suspended under the roofs on the side of the street.

Butumanklan, on the other hand literally means "anal wounds". It would be the relaxation of the muscles of the rectum. A patient suffering from this illness struggles to contain the bowel gas.

Plants indicated for the treatment of these diseases are certainly well known, but malaria and its associated symptoms (fever, convulsion, jaundice) were most cited. In fact, 21 species (18\% of all medicinal plants) are known for their use in the treatment of malaria. Among these species six are known only for this single indication: Alstonia boonei, Azadirachta indica A.Juss., Bambusa vulgaris Schard, Harungana madagascariensis, Spathodea campanulata P.Beauv. and Vernonia conferta. These species, which recorded a low use-value $(\mathrm{UV}=0.02)$ are in fact highly specialized in the treatment of a single disease. This poses the problem of interpretation of use-value in the case of studies in medicinal plants. If it is true that a high use-value of a plant indicates its importance within a community $[22,30]$, nevertheless, a UV close to 0 could indicate a high degree of specialization for a plant rather than a low use, when the frequency of citation of this plant is important. That is the case of Harungana madagascariensis which is widely known by Ehotile people as the anti-malarial plant.

This plant is used by other people of Côte d'Ivoire and Africa for the same indications, certainly because of its bright orange yellow latex (doctrine of signatures). It is used in the composition of many mixtures against jaundice in Côte d'Ivoire [11], Cameroon [43], southern Nigeria and Congo [45]. The stem bark contains several compounds such as anthracene, kenganone and kengathranol, anthronoïdes (harunmadagascarin A and B) having an important antioxidant activity [46]. 
Pharmacological works have confirmed the important traditional use of this plant in the treatment of malaria. Indeed, the stem bark extract exhibited significant antiprotozoan effects against Trichomonas and Plasmodium both in vivo and in vitro [47] and also exhibit antianaemic activity, and it's known that anaemia is a universal feature of malaria [48].

In contrast to this species highly specialized, some plants recorded higher use-values ( $U V>0.1$ ). These plants included Zanthoxylum gilletii (UV $=0.15 ; 8$ indications), Ocimum gratissimum (UV = 0.13; 7 indications), Eclipta prostrata (UV $=0.11 ; 6$ indications) and Paullinia pinnata $\mathrm{L}$. (UV $=0.2,6$ indications). Considered as panaceas, these species were variously used by the Ehotile. For example, Ocimum gratissimum is used for first intension in the treatment of several diseases (Convulsion, dysmenorrhoea, infant diarrhoea, gastritis, headaches). This plant is widely used in Côte d'Ivoire [12] and elsewhere in sub-Saharan Africa. For example, 7 indications for this plant are known in Ghana [49] and 11 indications in the south-eastern Nigeria [3]. Zanthoxylum gilletii is also widely known in Côte d'Ivoire for the diversity of its therapeutic indications $[11,35]$. The use of Paullinia pinnata in the treatment of malaria, haemorrhoids and sexual asthenia is not specific to Ehotile. In Benin, the plant is used to treat malaria and haemorrhoids, while in Mali and Togo, it is used against sexual asthenia. It is known now that antioxidant activity of the phenolic compounds contained in roots and leaves of this plant is the basis of its efficiency in the treatment of erectile dysfunction [50].

Other plants used by Ehotile are also known elsewhere or for similar purposes or for other pathologies. We will mention the example of Icacina mannii, Cassia occidentalis L., Baphia nitida, Phyllanthus amarus Schumach. \& Thonn., Picralima nitida and Momordica charantia.

The use of Icacina mannii against sexual asthenia seems not widespread. However, its use in the treatment of dysmenorrhoea and infertility in women (two conditions linked according to local experts) was raised in West Africa [51].

The leaves of Cassia occidentalis are used in the treatment of malaria in Ghana [37] and Nigeria [36], as used by Ehotile and phytochemical studies have shown the anti-malarial properties of this plant [52]. As used by Ehotile, the leaf juice of Baphia nitida is applied against parasitic skin diseases [53], but the use of this plant to cure lumbar pains was not found elsewhere. Phyllanthus amarus is widely used as a medicinal plant and as known by Ehotile. This plant is taken to facilitate childbirth and it is considered as a good tonic, diuretic and antipyretic [54].

Therapeutic Indications of Picralima nitida, widely known by Ehotile in the treatment of inguinal hernia, are reported in Côte d'Ivoire [11]. The akuammine, alkaloid abundant in the seeds can explain analgesic and relaxant action of this plant. Indeed, pharmacological studies revealed that the extract or isolated compounds from this species have analgesic, anti-inflammatory, hypoglyceamic, hypotensive, antiplasmodial, antimicrobial, antiulcer and antitumorigenic activities [55]. The anti-malarial use of Momordica charantia is widely known. Furthermore, in vitro anti-plasmodial activity has been demonstrated [56].

Finally, most of plants used as medicine by Ehotile are known by other people of Côte d'Ivoire and Africa. Indeed, 72 i.e. $62 \%$ of medicinal species identified are listed in the 304 highly pharmaceutical plants of Côte d'Ivoire [12]. However, some uses in traditional medicines of certain plants, as well as some therapeutic indications seem exclusive to Ehotile culture.

For example, the roots of Uvaria afzelii are employed in all medicinal preparations against hydrocephalus. Yet, this indication has not been reported and moreover, this disease is very rarely mentioned in the literature. Similarly, no mention of the use of Xylopia acutiflora's roots was found out. Nevertheless, these roots are widely known by Ehotile as sexual stimulants or as chewing sticks. Xylopia acutiflora is very known and appreciated by Ehotile women as chewing sticks as well for the pleasant minty sensation provided and the flexibility of the fibers obtained after chewing.

One of the issues raised in this survey is the availability of medicinal resources. As highlighted by various authors $[3,4]$, access to medicinal resources is an acute problem in Africa. Loss of natural vegetation, due to various causes, is also a major factor in the erosion of traditional knowledge [57]. Indeed, it is always considered that the resources of the deep forest are more effective than ruderal or introduced species. For example, Tetracera alnifolia abounds, but mature plants containing the sought exudate are only found in old marshland, that are scarce and difficult to access.

Yet, despite the deterioration of natural habitats, the use of medicinal plants is still alive among Ehotile. This situation of seemingly low-impact of environmental degradation on medicinal practices was also observed in Zambia [14]. However, we believe that this may be largely due to the fact that the plants commonly used are often on hand or in fallow or neighboring plantations.

Conversely, exploitation for medicinal purposes of certain plant species led to their scarcity or their disappearance [57]. The plant parts used and the way in which medicinal products are harvested also affect availability of the resources. Medicinal products, including barks, roots, and exudates, are widely used, but little is known about the sustainability of harvesting strategies currently employed [57]. Particularly vulnerable are those species occurring at low densities, those whose roots are harvested $[2,57]$. In our case, more than $50 \%$ of the 
dwindling species were sought for their roots, which should increase the pressure on these species.

Also vulnerable are those species whose bark or oil is extracted unsustainably [2] Harungana madagascariensis, Zanthoxylum gilletii and Icacina mannii are an illustration of unsustainable extraction, in our case. Harungana madagascariensis, as mentioned, is widely used as an anti-malarial, by specialized healers and housewives. Unfortunately, the stem bark, is easily removed and wholly extracted as well as leaves, leading to loss of the tree. According to the healers, currently, the plant only exists in the vicinity of a few marshy formations. Zanthoxylum gilletii is similar case but this species owes its survival to its resilience and its height. Icacina mannii is prized for its tuber, deemed effective against haemorrhoids and impotence. The collection of the whole tuber prevents any possibility of regeneration.

However, as noted elsewhere [57], the ecology of even the most widely used species is poorly understood and the impact of medicinal plant harvesting practices on the availability of resources are not known as well. Nevertheless, the relative impacts of harvesting medicinal plants are insignificant when compared with the impacts of deforestation [57].

\section{Conclusions}

The study of medicinal plants in Côte d'Ivoire covers many areas ranging from simple monographs to phytochemical and pharmacological studies. Few works have tried to estimate the knowledge about plants by a quantitative approach. This way of doing ethnobotany is in its early days in sub-Saharan Africa and should experience strong changes like what is done in Europe, Brazil and North America for example.

The study of the use of medicinal plants in Ehotile was interesting given their lifestyle (fishing) and especially the degradation of their environment.

The disappearance of natural formations in Ehotile land could indicate a low knowledge and use of medicinal plants by Ehotile. Rather, medicinal plants play an important role in the daily life of Ehotile thereby coming as complementary and alternative to modern health system. 123 plants were inventoried. They are used, in addition to medical prescriptions or exclusively, for treating several ailments most common of which are; malaria, sexual asthenia, hydrocephalus, pregnancy disorders and dysmenorrhea. Some of these plants are known for only one indication whereas, others have multiple usages.

The information was collected from two informants groups: the laypeople and key informants. Key informants helped us to confirm much of the information received from laypeople. The Informant Consensus Factor was only applied to this group and it was confirmed that they were, each highly specialized in the treatment of various diseases. With these local experts, the availability of identified plants was also defined. Some medicinal plants were easily accessible, whereas, others were scarce or no longer found in the region. It is feared that the scarcity of plants is a precursor to the disappearance of the rich knowledge and practices associated with them.

Two other aspects of this study are ongoing and should allow, with key informants, to specify the extent of the impact of environmental degradation on Ehotile medicinal practices firstly, and secondly to estimate the effect of medicinal plants harvesting on the availability of the most used species.

\section{Competing interests}

The authors declare that they have no competing interests.

\section{Authors' contributions}

MDF carried out the field ethnobotany research and drafted the manuscript. NDFR participated in the field research and handled to confirm the botanical determinations of the plants. KKL participated in study inception, performed the data analysis and participated to the draft of manuscript. All authors read and approved the final manuscript.

\section{Authors' information}

MDF, NDFR and KKL are assistant-lecturer at the nature Sciences' faculty of nangui abrogoua university, Abidjan. MDF is an ethnobotanist working on all forms of traditional uses of plants by the ancient peoples of Côte d'Ivoire and their strategies for plant conservation. NDFR is a specialist in systematic botany and works also on traditional use of plants. KKL is a specialist in African pharmacopoeia.

\section{Acknowledgements}

We are very grateful to Ms. Gloria K. BORONA, kagosi MWAMULOWE for the time they spent in reviewing the manuscript and for their valuable suggestions. We would also like to thank people of mélékoukro, akounougbé, assomlan and etuoboué for their openness and warm welcome.

Received: 6 September 2014 Accepted: 21 January 2015

Published online: 14 March 2015

\section{References}

1. Fasihuddin BA, Ghazally I. Medicinal plants used by Kadazandusun communities around Crocker Range. ASEAN Rev Environ Conserv (ARBEC). 2003:1:1-10.

2. Cunningham $A B$. Setting priorities at the interface between conservation and primary health care, People and Plants Working Paper 1. Paris: UNESCO; 1993.

3. Okafor J, Ham R. Identification, utilisation et conservation des plantes médicinales dans le Sud-est du Nigeria. Thèmes biodiv Afr. 1999;3:1-8.

4. Meunier $Q$, Bellefontaine R, Boffa JM. Le drageonnage pour la régénération d'espèces médicinales en Afrique tropicale : cas du Spathodea campanulata en Ouganda. VertigO. 2006;7(2):1-6.

5. Balick MJ, Cox PA. Plants, people, and culture, The Science of Ethnobotany. New York, USA: Scientific American Library; 1996.

6. Alexiades MN. Ethnobotany in the Third Millennium: expectations and unresolved issues. Delpinoa. 2003;45:15-28.

7. Dounias E, Rodrigues W, Petit C. Review of ethnobotanical literature for Central and West Africa. Bul Afric Ethnob Net. 2000:2:5-117.

8. Visser LE. Plantes médicinales de la Côte d'Ivoire. Une étude ethnobotanique des usages médicaux et comestibles des plantes sauvages par les Ando de la Côte d'Ivoire (Afrique occidentale). Wageningen: Mededelingen Landbouwhogeschool; 1975.

9. Lorougnon G. La médecine traditionnelle africaine: plantes et pharmacopée chez les Bété de la région de Daloa (Côte d'Ivoire). Abidjan, Côte d'Ivoire: Université Abidjan; 1993. 
10. N'guessan K. Les plantes médicinales et pratiques médicales traditionnelles chez les peuples Abbey et Krobou du Département d'Agboville (Côte d'Ivoire). PhD thesis. Abidjan: Université FHB Cocody, UFR Bioscience; 2008.

11. Bouquet A, Debray M. Plantes médicinales de la Côte d'Ivoire. Paris: Travaux et documents de l'ORSTOM; 1974.

12. Adjanohoun E, Aké Assi L. Contribution au recensement des plantes médicinales de Côte d'Ivoire. Abidjan: CNF; 1979.

13. World Health Organization. Côte d'Ivoire: Health profile, 2010. http://www. afro.who.int/index.php?option=com_docman\&task=doc_download\&gid= 7117\&ltemid=2593.

14. Adjemian M. Medicine from the forest: the impact of deforestation on medicinal plant availability and use in the Bilili Game Management Area, Southern Zambia. Thesis, McGill University, 2008.

15. Malan DF. Utilisations traditionnelles des plantes et perspective de cogestion des aires protégées de Côte d'Ivoire: cas du Parc National des lles Ehotilé (littoral est de la Côte d'Ivoire), PhD Thesis. Abidjan: University of Abobo-Adjamé, Nature Science Department; 2008.

16. Malan DF, Aké Assi L, Tra Bi FH, Neuba D. Diversité floristique du Parc National des lles Ehotilé (Littoral est de la Côte d'Ivoire). Bois For Trop. 2007;292(2):49-58.

17. Lucena RFP, Lima E, Albuquerque UP. Does the local availability of woody Caatinga plants (Northeastern Brazil) explain their use value? Econ Bot. 2007:61(4):347-61.

18. Guillaumet J-L, Adjanohoun E. La végétation. In: Avenard JM, Eldin M, Girard G, Sircoulon J, Touchebeuf P, Gillaumet JL, Adjanohoun E, Perraud A, editors. Le milieu naturel de la Côte d'Ivoire. Paris: ORSTOM; 1971.

19. Perrot $\mathrm{CH}$. Les Eotilé de Côte d'Ivoire auX XVIII ${ }^{e}$ et $X I X^{e}$ siècles. Pouvoir lignager et religion. Paris: Publications de la Sorbonne; 2008

20. Philips O, Gentry A. The useful woody plants of Tambopata, Peru: I. Statistical hypothesis tests with a new quantitative technique. Econ Bot. 1993:47:s16.

21. Hoffman B, Gallaher T. Importance Indices in Ethnobotany. Ethnobot Res Appl. 2007:5:201-18.

22. Albuquerque UP, Lucena RFP, Monteiro JM, Florentino ATN, Almeida CFCBR. Evaluating two quantitative ethnobotanical techniques. Ethnobot Res Appl. 2006;4:51-60.

23. Tardío J, Pardo-de-Santayana M. Cultural importance Indices: a comparative analysis based on the useful wild plants of Southern Cantabria (Northern Spain). Econ Bot. 2008;62(1):24-39.

24. Quinlan M. Considerations for collecting freelists in the field: examples from ethobotany. Field Methods. 2005;17(3):219-34.

25. Schrauf RW, Sanchez J. Using freelisting to identify, assess, and characterize age differences in shared cultural domains. Psychol Sci Soc Sci. 2008;63:385-93.

26. Smith JJ, Borgatti SP. Salience counts_and so does accuracy: correcting and updating a measure for free-list-item salience. J Ling Anthropol. 1998;7(2):208-9.

27. Sutrop U. List task and a cognitive salience index. Field Methods. 2001;13(3):263-76.

28. Rossato SC, Leitão-Filho $\mathrm{H}$, Begossi A. Ethnobotany of caiçaras of the Atlantic Forest Coast (Brasil). Econ Bot. 1999;53:387-95.

29. Heinrich M, Ankli A, Frei B, Weimann C, Sticher O. Medicinal plants in Mexico: Healers' consensus and cultural importance. Soc Sci Med. 1998;47:1863-75.

30. Gazzaneo LRS, Lucena RFP D, Albuquerque UP D. Knowledge and use of medicinal plants by local specialists in a region of Atlantic Forest in the state of Pernambuco (Northeastern Brazil). J Ethnobiol Ethnomed. 2005;1:9.

31. World Health Organization. International statistical classification of diseases and related health problems. Volume 2, Instruction manual. 2010 Edition. Geneva, Switzerland: WHO Library Cataloguing-in-Publication Data; 2011.

32. Ogbadoyi EO, Abdulganiy AO, Adama TZ, Okogun JI. In vivo trypanocida activity of Annona senegalensis Pers. leaf extract against Trypannosoma brucei brucei. J Ethnopharmacol. 2007;112(1):85-9.

33. Tra Bi FH, Koné MW, Kouamé NF. Antifungal activity of Erigeron floribundus (Asteraceae) from Côte d'Ivoire, West Africa. Trop J Pharm Res. 2008;7 (2):975-9.

34. Ajaiyeoba E, Falade M, Ogbole O, Okpako L, Akinboye D. In vivo antimalarial and cytotoxic properties of Annona senegalensis extract. Afr J Trad CAM. 2006;3(1):137-41

35. Zirihi GN, Datte JY, Kra-Adou KM, Grellier P. Phytochemical and pharmacologica studies of alcoholic extract of Fagara macrophylla (Oliv) Engl(Rutaceae):chemical structure of active compound inducing antipaludic activity. J Chinese Clin Med. 2007;2(4):205-10.
36. Singh $\mathrm{S}$, Singh R. Herbal medicinal treatment of Malaria in Aliero Local Government Area, Kebbi, Nigeria. J Med Plants Stud. 2014;2(2):117-26.

37. Asase A, Oteng-Yeboah AA, Odamtten GT, Simmonds MSJ. Ethnobotanical study of some Ghanaian anti-malarial plants. J Ethnopharmacol. 2005;99:273-9.

38. Ancolio C, Azas N, Mahiou V, Ollivier E, Di-Giorgio C, Keita A. Antimalarial activity of extracts and alkaloids isolated from six plants used in traditional medicine in Mali and Sao Tome. Phytother Res. 2002;16(7):646-9.

39. Ojewole JA. Hypoglycaemic effect of Clausena anisata (Willd) Hook methanolic root extract in rats. J Ethnopharmacol. 2002;81:231-7.

40. Ugochukwu NH, Babady NE. Antioxidant effects of Gongronema latifolium in hepatocytes of rat models of non-insulin dependent diabetes mellitus. Fitoterapia. 2002;73:612-8.

41. Yapo FA, Yapi FH, Ahiboh H, Hauhouot-Attounbre M-L, Guédé NZ, Djaman JA, et al. Immunomodulatory effect of the aqueous extract of Erigeron floribundus (Kunth) Sch Beep (Asteraceae) leaf in Rabbits. Trop J Pharm Res. 2011;10(2):187-93.

42. Lydia L, Lifongo LL, Simoben CV, Ntie-Kang F, Babiaka SB, Judson PN. A bioactivity versus ethnobotanical survey of medicinal plants from Nigeria. West Africa Nat Prod Bioprospect. 2014;4:1-19.

43. Betti JL. An ethnobotanical study of medicinal plants among the Baka Pygmies in the Dja Biosphere Reserve. Cameroon Afr Study Monogr. 2004;25(1):1-27.

44. Mukohya V, Kalau JM, Mutambwa M, Kakudji A. Appréhension de la maladie et dimension symbolique du traitement. In: Petit P, Mukohya V, editors. Bunganga Ya Mici, Guérisseurs et plantes médicinales à Lubumbashi. Lubumbashi: Université de Lubumbashi, Observatoire du changement urbain, CUD; 2004. p. 63-76.

45. Adjanohoun E, Ahyi MRA, Aké Assi L, Dramane K, Elewude JA, Fadoju SO, et al. Contribution to ethnobotanical and floristic studies in Western Nigeria. Paris: CSTR/OUA; 1991

46. Kouam SF, Ngadjui BT, Krohn K, Wafo P, Ajaz A, Choudhary MI. Prenylated anthronoid antioxidants from the stem bark of Harungana madagascariensis. Phytochemistry. 2005;66(10):1174-9.

47. Iwalewa EO, Omisore NO, Adewunmi CO, Gbolade AA, Ademowo OG, Nneji C, et al. Anti-protozoan activities of Harungana madagascariensis stem bark extract on trichomonads and malaria. J Ethnopharmacol. 2008;117(3):507-11.

48. Iwalewa EO, Omisore NO, Daniyan OM, Adewunmi CO, Taiwo BJ, Fatokun $\mathrm{OA}$, et al. Elemental compositions and anti-anemic property of Harungana madagascariensis stem bark. Bangladesh J Pharmacol. 2009;4:115-21.

49. Agbovie T, Amponsah K, Crentsil OR, Dennis F, Odamtten GT, Ofusohene-Djan W. Conservation and sustainable use of medicinal plants in Ghana: ethnobotanical survey. Cambridge UK: UNEP-WCMC; 2002.

50. Zamblé A, Carpentier M, Kandoussi A, Sahpaz S, Petrault O, Ouk T, et al. Paullinia pinnata extracts rich in polyphenols promote vascular relaxation via endothelium-dependent mechanisms. J Cardiovasc Pharmacol. 2006;47(4):599-608.

51. Burkill HM. The useful plants of west tropical Africa, vol. 2. 2nd ed. Kew, Richmond, UK: Families E-I, Royal Botanic Gardens; 1994.

52. Tona L, Cimanga RK, Mesia K, Musuamba CT, De Bruyne T, Apers S. In vitro antiplasmodial activity of extracts and fractions from seven medicinal plants used in the Democratic Republic of Congo. J Ethnopharmacol. 2004;93:27-32

53. Cardon D, Jansen PCM. Baphia nitida Lodd. In: Jansen PCM, Cardon D, editors. PROTA 3: Dyes and tannins [CD-Rom]. Wageningen, Netherlands: PROTA; 2005.

54. Oudhia P. Phyllanthus amarus Schumach. \& Thonn. [Internet]. In: GH, Gurib-Fakim A, editors. Record from Protabase Schmelzer. Wageningen, Netherlands: PROTA; 2008.

55. Erharuyi O, Falodun A, Langer P. Medicinal uses, phytochemistry and pharmacology of Picralima nitida (Apocynaceae) in tropical diseases: a review. Asian Pac J Trop Med. 2014;7(1):1-8.

56. Yousif SA. In vitro screening of antiplasmodium activity of momordica charantia. J Nat Resour Environ Stu. 2014;2(3):29-33.

57. Shanley $P, L$ Luz L. The impacts of forest degradation on medicinal plant use and implications for health care in Eastern Amazonia. Bioscience. 2003;53(6):573-84. 